\title{
Gaussian Quadrature Involving Einstein and Fermi Functions With an Application to Summation of Series*
}

\author{
By Walter Gautschi and Gradimir V. Milovanović
}

\begin{abstract}
Polynomials $\pi_{k}(\cdot)=\pi_{k}(\cdot ; d \lambda), k=0,1,2, \ldots$, are constructed which are orthogonal with respect to the weight distributions $d \lambda(t)=\left(t /\left(e^{t}-1\right)\right)^{r} d t$ and $d \lambda(t)=$ $\left(1 /\left(e^{t}+1\right)\right)^{r} d t, r=1,2$, on $(0, \infty)$. Moment-related methods being inadequate, a discretized Stieltjes procedure is used to generate the coefficients $\alpha_{k}, \beta_{k}$ in the recursion formula $\pi_{k+1}(t)=\left(t-\alpha_{k}\right) \pi_{k}(t)-\beta_{k} \pi_{k-1}(t), k=0,1,2, \ldots, \pi_{0}(t)=1, \pi_{-1}(t)=0$. The discretization is effected by the Gauss-Laguerre and a composite Fejér quadrature rule, respectively. Numerical values of $\boldsymbol{\alpha}_{k}, \boldsymbol{\beta}_{k}$, as well as associated error constants, are provided for $0 \leqslant k \leqslant 39$. These allow the construction of Gaussian quadrature formulae, including error terms, with up to 40 points. Examples of $n$-point formulae, $n=5(5) 40$, are provided in the supplements section at the end of this issue. Such quadrature formulae may prove useful in solid state physics calculations and can also be applied to sum slowly convergent series.
\end{abstract}

1. Introduction. We are interested in Gaussian quadrature on $[0, \infty]$ relative to the weight functions $\varepsilon_{r}(t)=\left(t /\left(e^{t}-1\right)\right)^{r}$ and $\varphi_{r}(t)=\left(1 /\left(e^{t}+1\right)\right)^{r}$. These functions arise, for example, in solid state physics and are referred to, when $r=1$, as Einstein and Fermi functions, respectively. Integrals with respect to the measure $d \lambda(t)=$ $\varepsilon_{r}(t) d t, r=1$ and 2 , are widely used in phonon statistics and lattice specific heats $[7, \S 10],[1, \S 2.4]$, and occur also in the study of radiative recombination processes [9, §9.2]. Specifically, the average energy of a quantum harmonic oscillator of frequency $\omega$ at temperature $T$ (representing thermal vibrations of crystal lattice atoms) is given by

$$
\bar{U}=\frac{\hbar \omega}{\exp (\hbar \omega / k T)-1}
$$

where $\hbar=h / 2 \pi$ ( $h$ is the Planck constant) and $k$ is the Boltzmann constant. Therefore, $\bar{U}=k T \varepsilon_{1}(\hbar \omega / k T)$, where $\varepsilon_{1}$ is Einstein's function. Letting $g(\omega)$ denote the phonon density of states function, and integrating (1.1) over the entire frequency range, the total energy of thermal vibration of the crystal lattice becomes

$$
U=\int_{0}^{\infty} \frac{\hbar \omega}{\exp (\hbar \omega / k T)-1} g(\omega) d \omega=\frac{k^{2} T^{2}}{\hbar} \int_{0}^{\infty} \varepsilon_{1}(t) g((k T / \hbar) t) d t
$$

Received April 11, 1983; revised November 14, 1983 and April 3, 1984.

1980 Mathematics Subject Classification. Primary 33A65, 65D32; Secondary 65A05, 65B10.

* The work of the first author was sponsored in part by the National Science Foundation under grant MCS-7927158A1. 
Furthermore, differentiating $U$ with respect to temperature $T$, yields the crystal lattice heat capacity at constant volume,

$$
C_{v}=\frac{\partial U}{\partial T}=\int_{0}^{\infty} \frac{(\hbar \omega / k T)^{2}}{(\exp (\hbar \omega / k T)-1)^{2}} k g(\omega) \exp (\hbar \omega / k T) d \omega,
$$

that is,

$$
C_{v}=\frac{k^{2} T}{\hbar} \int_{0}^{\infty} \varepsilon_{2}(t) f(t) d t \quad \text { where } f(t)=e^{t} g((k T / \hbar) t) .
$$

Similarly, integrals of the type $\int_{0}^{\infty} \varphi_{1}(t) f(t) d t$ (in fact, more generally $\int_{0}^{\infty} \varphi_{1}\left(t-t_{0}\right) f(t) d t$, for some parameter $\left.t_{0}\right)$ are encountered in the dynamics of electrons in metals, where they express the specific heat of the electron gas $[8, \S 9.17]$. For an application to heavy doped semiconductors, see also [10].

It will be shown in Section 4 that integration with respect to $d \lambda(t)=\varepsilon_{1}(t) d t$ and $d \lambda(t)=\varphi_{1}(t) d t$ is also useful in summing slowly convergent series whose general term is expressible in terms of a Laplace transform or its derivative.

Gaussian quadrature relative to the weight functions $\varepsilon_{r}$ and $\varphi_{r}$ will be particularly attractive in the case $r=1$, since both weight functions then have poles with nonzero residues, the former at $2 n \pi i, n= \pm 1, \pm 2, \ldots$, the latter at $(2 n+1) \pi i$, $n=0, \pm 1, \pm 2, \ldots$. Classical integration, even based on Gauss-Laguerre quadrature, will often be too slow, but will be used to generate the desired quadrature rules, at least in the case of the weight function $\varepsilon_{r}$; see (2.3) below.

The problem, basically, amounts to generating the coefficients $\alpha_{k}, \beta_{k}$ in the recursion formula

$$
\begin{gathered}
\pi_{k+1}(t)=\left(t-\alpha_{k}\right) \pi_{k}(t)-\beta_{k} \pi_{k-1}(t), \quad k=0,1,2, \ldots, \\
\pi_{-1}(t)=0, \quad \pi_{0}(t)=1
\end{gathered}
$$

for the (monic) orthogonal polynomials $\pi_{k}(\cdot)=\pi_{k}(\cdot ; d \lambda), k=0,1,2, \ldots\left(\beta_{0}\right.$ is arbitrary, but will be defined as $\beta_{0}=\int_{0}^{\infty} d \lambda(t)$.) Once the first $n$ of these coefficients, $\alpha_{k}, \beta_{k}, k=0,1, \ldots, n-1$, and $\beta_{n}$, are known, the $m$-point Gaussian quadrature formula

$$
\begin{gathered}
\int_{0}^{\infty} f(t) d \lambda(t)=\sum_{\mu=1}^{m} \lambda_{\mu} f\left(\tau_{\mu}\right)+R_{m}(f), \\
R_{m}(f)=\gamma_{m} f^{(2 m)}(\tau), \quad 0<\tau<\infty,
\end{gathered}
$$

including the error constant $\gamma_{m}$, can easily be obtained for any $m$ with $1 \leqslant m \leqslant n$. The nodes $\tau_{\mu}=\tau_{\mu}^{(m)}$, indeed, are the eigenvalues of the (symmetric tridiagonal) Jacobi matrix

$$
J_{m}=\left[\begin{array}{cccc}
\alpha_{0} & \sqrt{\beta_{1}} & & 0 \\
\sqrt{\beta_{1}} & \alpha_{1} & \sqrt{\beta_{2}} & \\
& \ddots & \ddots & \sqrt{\beta_{m-1}} \\
0 & & \sqrt{\beta_{m-1}} & \alpha_{m-1}
\end{array}\right],
$$


while the weights $\lambda_{\mu}=\lambda_{\mu}^{(m)}$ are given by $\lambda_{\mu}=\beta_{0} v_{\mu, 1}^{2}$ in terms of the first components $v_{\mu, 1}$ of the corresponding normalized eigenvectors; see, e.g., $[3, \S 5.1]$. Moreover,

$$
\gamma_{m}=\frac{\beta_{0} \beta_{1} \cdots \beta_{m}}{(2 m) !}, \quad m=1,2, \ldots, n .
$$

A number of methods are available, and have been analyzed in [4], for computing the recursion coefficients $\alpha_{k}, \beta_{k}$. They are either based on the moments (or modified moments) of the weight distribution $d \lambda(t)$, or else use a suitable discretization of the inner product

$$
(u, v)=\int_{0}^{\infty} u(t) v(t) d \lambda(t), \quad u, v \in \mathbf{P}
$$

where $u, v$ are polynomials. In the next section we discuss the relative merits of these methods for constructing the particular orthogonal polynomials at hand.

2. Generation of the Recursion Coefficients. We consider first the weight function $\varepsilon_{r}$. The classical approach (for example, the Chebyshev algorithm; see [4, §2.3]) departs from the moments of the weight function, which, in the case at hand, can be expressed in terms of the Riemann zeta function $\zeta(z)$,

$$
\begin{aligned}
& \mu_{k}=\int_{0}^{\infty} t^{k} \varepsilon_{r}(t) d t \\
& =\left\{\begin{array}{l}
(k+1) ! \zeta(k+2), \quad r=1, \\
(k+2) ![\zeta(k+2)-\zeta(k+3)], \quad r=2,
\end{array} \quad k=0,1,2, \ldots .\right.
\end{aligned}
$$

It is well-known, however, that the moments $\mu_{k}$ define the recursion coefficients $\alpha_{k}$, $\beta_{k}$ and the associated Gaussian quadrature formulae poorly in a numerical sense. The map $G_{n}$ from the first $2 n$ moments $\mu_{k}, k=0,1,2, \ldots, 2 n-1$, to the weights $\lambda_{\nu}^{(n)}$ and nodes $\tau_{\nu}^{(n)}$ of the $n$-point Gaussian formula, in fact becomes progressively more ill-conditioned as $n$ increases. This is illustrated in Table 2.1, where in the second and third column the condition number of this map (in the sense of [4, Eq. (3.11)f]) is shown as a function of $n$ for $r=1$ and $r=2$, respectively. (Numbers in parentheses indicate decimal exponents.)

\section{TABLE 2.1}

The numerical condition of various maps relating to the construction of orthogonal polynomials $\pi_{k}\left(\cdot ; \varepsilon_{r} d t\right), r=1,2$.

\begin{tabular}{cccc|ccc}
$n$ & $\begin{array}{c}\text { cond } G_{n} \\
r=1\end{array}$ & $\begin{array}{c}\text { cond } G_{n} \\
r=2\end{array}$ & $\begin{array}{c}\text { cond } \tilde{G}_{n} \\
r=1\end{array}$ & $n$ & $\begin{array}{c}\text { cond } H_{n} \\
r=1\end{array}$ & $\begin{array}{c}\text { cond } H_{n} \\
r=2\end{array}$ \\
\hline 3 & $1.686(2)$ & $7.620(1)$ & $1.415(1)$ & 5 & $7.665(0)$ & $7.980(0)$ \\
6 & $1.288(5)$ & $7.171(4)$ & $2.934(3)$ & 10 & $2.163(1)$ & $2.161(1)$ \\
9 & $9.092(7)$ & $5.820(7)$ & $9.795(5)$ & 15 & $3.299(1)$ & $3.356(1)$ \\
12 & $6.334(10)$ & $4.471(10)$ & $4.039(8)$ & 20 & $4.256(1)$ & $4.244(1)$ \\
15 & $4.409(13)$ & $3.344(13)$ & $1.878(11)$ & 30 & $6.761(1)$ & $6.783(1)$ \\
18 & $3.076(16)$ & $2.465(16)$ & $9.441(13)$ & 40 & $8.531(1)$ & $8.556(1)$
\end{tabular}


Sometimes the condition can be improved by employing modified moments $\nu_{k}=\int_{0}^{\infty} p_{k}(t) \varepsilon_{r}(t) d t$, where $\left\{p_{k}\right\}$ is a system of (monic) polynomials suitably chosen. If the support interval is infinite, however, as in the case at hand, the improvement is often not sufficient to make this a viable alternative. The fourth column of Table 2.1 indeed confirms this. Here, $r=1$, and $\varepsilon_{1}(t) \sim t e^{-t}$ as $t \rightarrow \infty$, so that the monic Laguerre polynomials with parameter $\alpha=1$,

$$
p_{k}(t)=(-1)^{k} k ! L_{k}^{(1)}(t), \quad k=0,1,2, \ldots
$$

appear to be the most natural choices for the polynomials $p_{k}$. The corresponding modified moments can be shown to be expressible in terms of the (forward) differences, with unit step, of the Riemann zeta function at $z=2$,

$$
\nu_{k}=\int_{0}^{\infty} p_{k}(t) \varepsilon_{1}(t) d t=\left.(k+1) ! \Delta^{k} \zeta(z)\right|_{z=2}, \quad k=0,1,2, \ldots
$$

The map $\tilde{G}_{n}$ in Table 2.1 is the map from the first $2 n$ normalized modified moments $\tilde{\nu}_{k}=\nu_{k}\left[\int_{0}^{\infty} p_{k}^{2}(t) t e^{-t} d t\right]^{-1 / 2}, k=0,1, \ldots, 2 n-1$, to the weights $\lambda_{\nu}^{(n)}$ and nodes $\tau_{\nu}^{(n)}$ of the $n$-point Gaussian quadrature formula (1.4) (with $d \lambda(t)=\varepsilon_{1}(t) d t$ ). The entries in the fourth column exhibit the estimate derived in [4, Eq. (3.15)] for the condition of $\tilde{G}_{n}$. It can be seen that cond $\tilde{G}_{n}$, while somewhat smaller than cond $G_{n}$, is still growing unacceptably fast.

The modified Chebyshev algorithm (cf. $[4, \$ 2.4]$ ), run in single precision on the CDC 6500 (machine precision: ca. 15 significant decimal digits), with the modified moments (2.2) computed in double precision, indeed loses accuracy very much in accordance with the growth of cond $\tilde{G}_{n}$; the number of correct decimal digits observed in $\alpha_{k}, \beta_{k}, k=3(3) 18$, is $14,11,10,6,2,0$, respectively, not a single digit being correct for $k=18$, not even the sign of $\beta_{18}$.

An additional complication arises in connection with the high-order differences in (2.2), which are subject to considerable cancellation errors. For $k=40$, for example, one must expect a loss of approx. 12 decimal digits.

In contrast, methods based on discretization appear to be incomparably more effective, particularly if one uses the natural discretization based on the GaussLaguerre quadrature rule,

$$
\begin{gathered}
\int_{0}^{\infty} p(t)\left(\frac{t}{e^{t}-1}\right)^{r} d t=\frac{1}{r} \int_{0}^{\infty} p(t / r)\left(\frac{t / r}{1-e^{-t / r}}\right)^{r} e^{-t} d t \\
\approx \frac{1}{r} \sum_{k=1}^{N} \lambda_{k}^{L} p\left(\tau_{k}^{L} / r\right)\left(\frac{\tau_{k}^{L} / r}{1-e^{-\tau_{k}^{L} / r}}\right)^{r}, \quad p \in \mathbf{P} .
\end{gathered}
$$

Here, $\tau_{k}^{L}$ are the zeros of the Laguerre polynomial of degree $N$, and $\lambda_{k}^{L}$ the associated Christoffel numbers. The discretized Stieltjes procedure (cf. [4, §2.2]), based on (2.3), then converges fairly rapidly as $N \rightarrow \infty$. For example, to obtain the first 12 recursion coefficients $(n=12)$ to 12 correct decimals [ 25 correct decimals] requires $N=49[N=127]$, when $r=1$, and $N=41[N=85]$, when $r=2$. The first 40 coefficients $(n=40)$ can be obtained accurately to 25 decimal digits with $N=281$ for $r=1$ and $N=201$ for $r=2$. Also the numerical stability of the procedure, 
which is roughly determined (cf. $[4, \S 3.4])$ by the condition of the map

$$
H_{n}:\left(\lambda_{1}, \ldots, \lambda_{n}, \tau_{1}, \ldots, \tau_{n}\right) \rightarrow\left(\alpha_{0}, \ldots, \alpha_{n-1}, \beta_{0}, \ldots, \beta_{n-1}\right),
$$

is rather good. This can be seen from the last two columns of Table 2.1, which display the condition number of $H_{n}$ in the sense of [4, Eq. (3.8)f].

One might expect that a discretization analogous to the one in (2.3) works well also for the weight function $\varphi_{r}$. This, however, is not the case, at least not if $r=1$. Convergence turns out to be slow on account of the poles at $\pm i \pi$, which are twice as close to the real axis as in the case of $\varepsilon_{1}$. For example, to obtain 20 correct decimal digits (in the case $r=1$ and for $n=40$ ), requires $N=361$. We used instead a discretization procedure based on the composite Fejér quadrature rule, decomposing the interval of integration into four subintervals, $[0, \infty]=[0,10] \cup[10,100] \cup$ $[100,500] \cup[500, \infty]$. Using $N=201$ for $r=1$, and $N=281$ for $r=2$, on each subinterval, then yields the first $n=40$ recursion coefficients accurately to 25 decimal digits. The same procedure (with $n=50$ and $N=251$ ) was also used to check the computation based on (2.3) for $\varepsilon_{r}, r=1,2$. The maximum discrepancy observed was 1 unit in the 25 th digit.

3. Numerical Results. Using the procedures outlined in the last two paragraphs of Section 2, we obtained the results shown in Tables 1-4 of the Appendix. The coefficients $\beta_{0}=\zeta(2)=\pi^{2} / 6$ in Table 1 and $\beta_{0}=2[\zeta(2)-\zeta(3)]$ in Table 2 (cf. Eq. (2.1)), agree to all 25 decimal digits with the values obtained from the 41S-table of the Riemann zeta function in [6]. Similarly, one checks the values $\beta_{0}=\ln 2$ in Table 3 and $\beta_{0}=\ln 2-\frac{1}{2}$ in Table 4. Corresponding $n$-point Gaussian quadrature formulae, $n=5(5) 40$, for $r=1$ and $r=2$, can be found to 25 significant decimal digits in Tables 5-8 in the supplements section at the end of this issue.

We illustrate these formulae by computing the integrals

$$
\begin{aligned}
& I_{1}=\int_{0}^{\infty} e^{-t} \frac{t}{e^{t}-1} d t=\zeta(2)-1 \\
& I_{2}=\int_{0}^{\infty} e^{-t}\left(\frac{t}{e^{t}-1}\right)^{2} d t=2[\zeta(2,2)-2 \zeta(3,2)], \\
& I_{3}=\int_{0}^{\infty} e^{-t} \frac{d t}{e^{t}+1}=1-\ln 2 \\
& I_{4}=\int_{0}^{\infty} e^{-t} \frac{d t}{\left(e^{t}+1\right)^{2}}=\frac{3}{2}-2 \ln 2
\end{aligned}
$$

where $\zeta(z, a)$ is the generalized zeta function. Table 3.1 shows the $n$-point approximations $I_{i}(n)$ to $I_{i}, i=1,2$, together with the relative errors $r_{i}(n)$, for $n=5(5) 25$. Similarly, $I_{i}(n)$ and $r_{i}(n), i=3,4$, are shown in Table 3.2. In each entry the first digit in error is underlined. The error term in (1.4) yields the bounds $\gamma_{n} / I_{i}$, $i=1,2,3,4$, for the relative error, which can be computed with the help of Tables 1-4 of the Appendix and the limit values in Tables 3.1, 3.2; they are summarized in Table 3.3 for $n=5(5) 25$. The bounds for $I_{2}, I_{4}$ are seen to be considerably sharper than those for $I_{1}, I_{3}$. 
TABLE 3.1

Gaussian approximations of the integrals $I_{1}$ and $I_{2}$ and relative errors.

\begin{tabular}{cllll}
$n$ & \multicolumn{1}{c}{$I_{1}(n)$} & $r_{1}(n)$ & \multicolumn{1}{c}{$I_{2}(n)$} & $r_{2}(n)$ \\
\hline 5 & $.644 \underline{7} 42$ & $3.0(-4)$ & $.4816 \underline{385}$ & $4.1(-6)$ \\
10 & $.6449340 \underline{5} 94$ & $1.1(-8)$ & $.48164052105 \underline{73} 7$ & $1.5(-12)$ \\
15 & $.644934066848 \underline{12} 17$ & $3.2(-13)$ & $.481640521058075731 \underline{12} 84$ & $3.3(-19)$ \\
20 & $.64493406684822643 \underline{1} 31$ & $8.0(-18)$ & $.481640521058075731345877 \underline{6}$ & $1.1(-25)$ \\
25 & $.64493406684822643647 \underline{72}$ & $1.8(-22)$ & &
\end{tabular}

TABLE 3.2

Gaussian approximations of the integrals $I_{3}$ and $I_{4}$ and relative errors.

\begin{tabular}{cllll}
$n$ & \multicolumn{1}{c}{$I_{3}(n)$} & $r_{3}(n)$ & \multicolumn{1}{c}{$I_{4}(n)$} & \multicolumn{1}{c}{$r_{4}(n)$} \\
\hline 5 & $.3068 \underline{1} 71$ & $1.2(-4)$ & $.113705 \underline{5} 28$ & $9.7(-7)$ \\
10 & $.30685281 \underline{8} 54$ & $2.9(-9)$ & $.113705638880 \underline{0} 91$ & $1.5(-13)$ \\
15 & $.3068528194400 \underline{3} 58$ & $6.2(-14)$ & $.11370563888010938116 \underline{32} 2$ & $2.0(-20)$ \\
20 & $.306852819440054690 \underline{2} 08$ & $1.2(-18)$ & .1137056388801093811655358 & $6.1(-26)$ \\
25 & $.30685281944005469058276 \underline{07}$ & $2.3(-23)$ & &
\end{tabular}

TABLE 3.3

Relative error bounds for $I_{i}(n), i=1,2,3,4$.

\begin{tabular}{cllll}
$n$ & $\gamma_{n} / I_{1}(n)$ & $\gamma_{n} / I_{2}(n)$ & $\gamma_{n} / I_{3}(n)$ & $\gamma_{n} / I_{4}(n)$ \\
\hline 5 & $4.8(-2)$ & $8.3(-5)$ & $1.2(-2)$ & $1.3(-5)$ \\
10 & $1.1(-4)$ & $3.0(-10)$ & $1.6(-5)$ & $1.9(-11)$ \\
15 & $1.9(-7)$ & $6.7(-16)$ & $2.0(-8)$ & $2.3(-17)$ \\
20 & $2.7(-10)$ & $1.2(-21)$ & $2.3(-11)$ & $2.5(-23)$ \\
25 & $3.6(-13)$ & $1.8(-27)$ & $2.5(-14)$ & $2.7(-29)$
\end{tabular}

4. Summation of Series. As an application of Gaussian integration with weight functions $\varepsilon_{1}$ and $\varphi_{1}$, we consider the summation of series whose general term is expressible in terms of the derivative of a Laplace transform, or in terms of the Laplace transform itself. Suppose, for example, that $a_{k}=-F^{\prime}(k)$, where

$$
F(p)=\int_{0}^{\infty} e^{-p t} f(t) d t, \quad \operatorname{Re} p \geqslant 1
$$

Then

$$
\sum_{k=1}^{\infty} a_{k}=\sum_{k=1}^{\infty} \int_{0}^{\infty} t e^{-k t} f(t) d t=\int_{0}^{\infty} \frac{t}{e^{t}-1} f(t) d t
$$

that is,

$$
-\sum_{k=1}^{\infty} F^{\prime}(k)=\int_{0}^{\infty} f(t) \varepsilon_{1}(t) d t .
$$

Similarly, for “alternating” series, one obtains

$$
-\sum_{k=1}^{\infty}(-1)^{k-1} F^{\prime}(k)=\int_{0}^{\infty} f(t) t \varphi_{1}(t) d t
$$


and

$$
\sum_{k=1}^{\infty}(-1)^{k-1} F(k)=\int_{0}^{\infty} f(t) \varphi_{1}(t) d t
$$

If the series on the left are slowly convergent and the respective function $f$ on the right is smooth, then low-order Gaussian quadrature applied to the integrals on the right provides one (among many other) possible summation procedure. If $f$ exhibits singularities, then the weight function may have to be modified if the effectiveness of the procedure is to be preserved (cf. Example 4.4). In the following examples, "(a)", “(b)", “(c)" refers to a, b, c of Eq. (4.2).

Example 4.1. (a) $\sum_{k=1}^{\infty}(k+1)^{-2}=\pi^{2} / 6-1$. Here, $-F^{\prime}(p)=(p+1)^{-2}$, and $F(p)=(p+1)^{-1}$, the integration constant being zero on account of $F(p) \rightarrow 0$ as $p \rightarrow \infty$. Thus, $f(t)=e^{-t}$, and the second column of Table 3.1 shows the rapid convergence of Gaussian quadrature in this case. For example, 15-point quadrature already yields 12 correct decimal digits. In contrast, 10000 terms of the series would give only 3-digit accuracy.

(b) $\sum_{k=1}^{\infty}(-1)^{k-1}(k+1)^{-2}=1-\pi^{2} / 12$. Applying Gaussian quadrature to the integral in (4.2b) yields the results shown in Table 4.1. To guarantee, on the basis of Leibniz' convergence criterion, the same accuracy as the one achieved for $n=15$ would require the summation of approximately 690000 terms.

(c) $\sum_{k=1}^{\infty}(-1)^{k-1}(k+1)^{-1}=1-\ln 2$. The convergence behavior of Gaussian quadrature applied to the integral in (4.2c) is evident from the second column of Table 3.2.

TABLE 4.1

Gaussian approximations $K(n)$ of the integral $K=\int_{0}^{\infty} e^{-t} t\left(e^{t}+1\right)^{-1} d t$ and relative errors.

\begin{tabular}{cll}
$n$ & \multicolumn{1}{c}{$K(n)$} & \multicolumn{1}{c}{$r(n)$} \\
\hline 5 & $.177 \underline{7} 53$ & $1.2(-3)$ \\
10 & $.1775329 \underline{7} 80$ & $6.5(-8)$ \\
15 & $.17753296657 \underline{6} 25$ & $2.1(-12)$ \\
20 & $.1775329665758867 \underline{915}$ & $5.5(-17)$ \\
25 & $.17753296657588678176 \underline{4027}$ & $1.3(-21)$ \\
30 & .177532966575886781763792 & $1.4(-25)$
\end{tabular}

Example 4.2. $F(p)=p^{-1} \exp (-1 / p),-F^{\prime}(p)=(p-1) p^{-3} \exp (-1 / p)$. The original function is here $f(t)=J_{0}(2 \sqrt{t})$. This being an entire function, we expect Gaussian quadrature in (4.2) to converge rapidly. This is confirmed in Table 4.2, which shows the relative errors for the $n$-point formula, $n=2(2) 12$. The exact sums (to 24 significant digits), as determined by Gaussian quadrature, are, respectively,

$$
\begin{aligned}
& \sum_{k=1}^{\infty}(k-1) k^{-3} \exp (-1 / k)=.342918943844609780961838, \\
& \sum_{k=1}^{\infty}(-1)^{k-1}(k-1) k^{-3} \exp (-1 / k)=-.0441559381340836052736928, \\
& \sum_{k=1}^{\infty}(-1)^{k-1} k^{-1} \exp (-1 / k)=.197107936397950656955672 .
\end{aligned}
$$


The first 10000 terms of the series yield, respectively, 3,7 and 4 correct decimal digits. The Bessel function $J_{0}$ was evaluated by means of the rational approximations in [5] indexed 5852, 6553 and 6953.

\section{TABLE 4.2}

Relative errors in Gaussian approximation of the integrals in $(4.2, \mathrm{a}-\mathrm{c})$, where $F(p)=p^{-1} \exp (-1 / p)$.

\begin{tabular}{clll}
$n$ & $(\mathrm{a})$ & \multicolumn{1}{c}{ (b) } & \multicolumn{1}{c}{ (c) } \\
\hline 2 & $4.48(-2)$ & $8.95(-1)$ & $1.77(-2)$ \\
4 & $3.80(-6)$ & $2.39(-4)$ & $9.65(-7)$ \\
6 & $3.35(-11)$ & $3.71(-9)$ & $6.32(-12)$ \\
8 & $7.01(-17)$ & $1.13(-14)$ & $1.05(-17)$ \\
10 & $6.86(-23)$ & $1.08(-20)$ & $1.27(-23)$ \\
12 & & $1.93(-23)$ &
\end{tabular}

Example 4.3. $F(p)=\left(1+p^{2}\right)^{-1 / 2},-F^{\prime}(p)=p\left(1+p^{2}\right)^{-3 / 2}$. Here, $f(t)=$ $J_{0}(t)$-again an entire function. Convergence of Gaussian quadrature in (4.2), while still satisfactory, is not quite as fast as in Example 4.2, presumably since the argument of $J_{0}$ is now essentially squared. The relative errors are shown in Table 4.3. For reference, we list the exact values of the respective sums:

$$
\begin{aligned}
& \sum_{k=1}^{\infty} k\left(1+k^{2}\right)^{-3 / 2}=.900524735348125924300853 \\
& \sum_{k=1}^{\infty}(-1)^{k-1} k\left(1+k^{2}\right)^{-3 / 2}=.234771442466894018686113 \\
& \sum_{k=1}^{\infty}(-1)^{k-1}\left(1+k^{2}\right)^{-1 / 2}=.440917473865185397183787
\end{aligned}
$$

The first 10000 terms yield 3,8 and 4 correct decimal digits, respectively.

TABLE 4.3

Relative errors in Gaussian approximation of the integrals in $(4.2, \mathrm{a}-\mathrm{c})$, where $F(p)=\left(1+p^{2}\right)^{-1 / 2}$.

\begin{tabular}{clll}
$n$ & \multicolumn{1}{c}{$(\mathrm{a})$} & \multicolumn{1}{c}{$(\mathrm{b})$} & \multicolumn{1}{c}{$(\mathrm{c})$} \\
\hline 5 & $1.68(-3)$ & $8.15(-3)$ & $1.99(-4)$ \\
10 & $8.03(-7)$ & $4.75(-6)$ & $2.11(-7)$ \\
15 & $4.05(-10)$ & $2.99(-9)$ & $9.01(-12)$ \\
20 & $1.64(-13)$ & $7.61(-13)$ & $2.26(-14)$ \\
25 & $5.79(-17)$ & $5.64(-16)$ & $2.52(-19)$ \\
30 & $2.69(-20)$ & $8.25(-20)$ & $2.33(-21)$ \\
35 & $5.11(-24)$ & $8.01(-23)$ & $3.88(-25)$
\end{tabular}


Example 4.4. $F(p)=2 p^{-1}(p+1)^{-1 / 2},-F^{\prime}(p)=(3 p+2) p^{-2}(p+1)^{-3 / 2}$. Here we have an example in which the original function, $f(t)=2 \operatorname{erf} \sqrt{t}$, is no longer smooth, having a square root singularity at $t=0$.

\section{TABLE 4.4}

Relative errors in Gaussian approximation of the integrals in $(4.2, \mathrm{a}-\mathrm{c})$, where

$F(p)=2 p^{-1}(p+1)^{-1 / 2}$, using weight functions $t^{1 / 2} \varepsilon_{1}(t)$ and $t^{1 / 2}{ }^{1 / 2}$
$n$

This severely retards convergence of Gaussian quadrature in (4.2). When $n=40$, for example, the relative errors are still $4.29(-4), 7.19(-6)$ and $4.80(-4)$, respectively. Better results can be obtained by integrating with respect to the measures $t^{1 / 2} \varepsilon_{1}(t) d t$ and $t^{1 / 2} \varphi_{1}(t) d t$. The recursion coefficients for the corresponding orthogonal polynomials have been computed by a discretized Stieltjes procedure $[4, \S 2.2]$ involving two different discretizations, one on the interval $[0,10]$, where a Gauss-Jacobi quadrature with parameters $\alpha=0, \beta=\frac{1}{2}$ (to account for the $t^{1 / 2}$-singularity at $t=0$ ) was used, and one on the interval $[10, \infty]$, where we used Gauss-Laguerre quadrature as before. With the special Gaussian quadrature rules thus obtained, convergence of the summation process is very satisfactory; see Table 4.4 . The exact values of the sums are:

$$
\begin{aligned}
& \sum_{k=1}^{\infty}(3 k+2) k^{-2}(k+1)^{-3 / 2}=2.571949632310480570278028 \\
& \sum_{k=1}^{\infty}(-1)^{k-1}(3 k+2) k^{-2}(k+1)^{-3 / 2}=1.485761529223412110869727 \\
& \sum_{k=1}^{\infty}(-1)^{k-1} 2 k^{-1}(k+1)^{-1 / 2}=1.039526533711568982971620
\end{aligned}
$$

The error function was evaluated by a double precison version of the incomplete gamma function routine in [2], observing that erf $\sqrt{t}=g^{*}\left(\frac{1}{2}, t\right)$ in the notation of [2].

Acknowledgment. The authors are indebted to Professors R. Colella and N. D. Stojadinović for discussions of solid state physics applications and for the references [1], [7]-[10]. 
Appendix A1: Recursion coefficients $\alpha_{k}, \beta_{k}$ and error constants $\gamma_{k}$ for orthogonal polynomials $\pi_{k}\left(\cdot ; \varepsilon_{r} d t\right), r=1,2$.

TABLE 1

Recursion coefficients and error constants in the case $r=1$

k

alpha(k) beta $(k)$

$1.644934066848226436472415 d+00$ $1.811783690642112489289654 d+00$ $5.676288706260277787680856 \mathrm{~d}+00$ $1.156744408657869148844530 d+01$ $1.947389445016451747566531 d+01$ $2.939056708923949547049877 d+01$ 4. $131469329707496049141402 d+01$ $5.524456680862040935918879 d+01$ $7.117904800086908599819680 d+01$ $8.911733086545634278259615 d+01$ $1.090588203798980126014925 d+02$ 1. $310030623930625789347323 d+02$ $1.549497009045172820025340 d+02$ $1.808984506906222174100004 d+02$ 2. $088490790318381268700652 d+02$ $2.388013930944475277549535 d+02$ 2. $707552309641486213037546 \mathrm{~d}+02$ $3.047104551169241967842131 d+02$ $3.406669475630502474295334 d+02$ $3.786246061681214841053902 d+02$ $4.185833418200242159627432 d+02$ 4. $605430762155397308587299 d+02$ $5.045037401085301054018693 d+02$ $5.504652719072102149716323 d+02$ $5.984276165390506043752540 d+02$ $6.483907245234129654656713 d+02$ $7.003545512072496003149494 d+02$ $7.543190561301266397056653 d+02$ $8.102842024927851127149421 d+02$ $8.682499567093196335570932 d+02$ 9. $282162880274321890289169 d+02$ $9.901831682045221630508258 d+02$ $1.054150571229892590247571 d+03$ $1.120118473085291539833540 d+03$ $1.188086851537513352077144 d+03$ $1.258055685957963670922452 d+03$ 1. $330024957165022879643043 d+03$ $1.403994647285782417304167 d+03$ $1.479964739634320690278091 d+03$ 1. $557935218604162422993492 d+03$ $\operatorname{gamma}(k)$

$1.6449 d+00$ $1.4901 d+00$ $7.0487 \mathrm{~d}-01$ 2. $7178 d-01$ $9.4512 \mathrm{~d}-02$ 3. $0864 d-02$ $9.6602 d-03$ $2.9323 d-03$ 8. $6965 d-04$ $2.5327 d-04$ 7. 2688d-0 5 2.0611d-0 5 5. $7857 d-06$ 1. $6102 d-06$ 4. $4482 \mathrm{~d}-07$ 1. $2210 d-07$ 3. $3325 d-08$ 9.0503d-09 2. 4469d-09 $6.5894 d-10$ 1. $7681 \mathrm{~d}-10$ 4.7287d-11 1. $2609 d-11$ 3.3531d-12 8.8944d-13 2. $3539 d-13$ $6.2163 d-14$ 1. $6384 \mathrm{~d}-14$ 4. 3103d-15 1. $1320 d-15$ 2. $9682 d-16$ $7.7711 d-17$ 2. $0317 \mathrm{~d}-17$ 5. $3049 \mathrm{~d}-18$ 1. $3834 d-18$ 3. $6032 d-19$ $9.3747 d-20$ 2. $4365 d-20$ 6. 3263d-21 1. $6410 \mathrm{~d}-21$ 
TABLE 2

Recursion coefficients and error constants in the case $r=2$

$0 \quad 8.110623843271969462056717 d-01$ $2.082489563360933113678297 d+00$ 3. $180472732492342276049514 d+00$ $4.230464687232567728899659 d+00$ $5.261814180222519507249016 d+00$ 6. $283970335641423071715338 d+00$ $7.300777826737402137770257 d+00$ 8. $314124893536196732880721 d+00$ 9. $325070412859877386826831 d+00$ $1.033426456179618464586238 d+01$ $1.134213320133091970071013 d+01$ $1.234896912257135049629721 d+01$ $1.335498141987434463665121 d+0$ $1.436032407203413184648565 d+01$ l. $536511338808887561282313 d+01$ $1.636943912406203612259834 d+0$ $1.737337182454617157330816 d+01$ $1.837696782017803014429121 d+01$ $1.938027271912508051564886 \mathrm{~d}+0$ $2.038332390222895035094720 d+01$ $2.138615234191184776508271 d+01$ $2.238878395166622155008636 \mathrm{~d}+0$ $2.339124060312416766916321 d+01$ $2.439354090348347602881194 d+01$ $2.539570079737826452062748 d+01$ $2.639773403826193082384386 d+01$ $2.739965256151148169598840 d+01$ $2.840146678261384239170508 d+01$ $2.940318583760677307621679 d+01$ 3. $040481777855517934134407 d+01$ 3. $140636973368393222174353 d+0$ 3. $240784803948646086438457 d+01$ 3. $340925835043190608386017 d+01$ 3. $441060573062978667260308 d+01$ $3.541189473086012145546484 d+01$ $3.641312945365461559438722 d+01$ $3.741431360856105438919087 \mathrm{~d}+01$ $3.841545055929548135777313 d+01$ $3.941654336415387638822144 d+01$ $4.041759481079403079082453 d+01$
$8.857543273772643021453540 d-01$ $5.721932339461376964598358 d-01$ $1.759962195010899092957389 d+00$ $3.452384919203117354550957 d+00$ $5.646769389936900623699487 d+00$ $8.344355062173523581471496 d+00$ 1. $154523064520239591047982 d+01$ 1. $524904924910513826966172 d+01$ $1.945541436638288259572407 d+01$ $2.416397877514391584300045 d+01$ $2.937445596792765549373146 d+01$ 3. $508661182861105008895423 d+01$ 4. $130025409989901518132003 d+01$ $4.801522341847326505433396 d+01$ $5.523138632084691795947509 d+01$ $6.294862990488389566003207 d+01$ $7.116685775941257898687472 d+01$ $7.988598684014213308377918 d+01$ $8.910594504902261454319077 d+01$ $9.882666933905573379469390 d+01$ $1.090481042149328176517281 d+02$ $1.197702005348084683811837 d+02$ 1. $309929145435295611827254 d+02$ $1.427162070855731132915232 d+02$ l. $549400429588849232857666 d+02$ $1.676643903802234140414907 d+02$ $1.808892205395246706020251 d+02$ $1.946145072259288312221530 d+02$ $2.088402265119435652344011 d+02$ $2.235663564851183557323020 d+02$ $2.387928770188127033667876 \mathrm{~d}+02$ $2.545197695753403827498683 d+02$ 2. $707470170360892004885838 d+02$ $2.874746035542450893092687 d+02$ 3. $047025144265597523463770 d+02$ 3. $224307359812435482146968 d+02$ $3.406592554795780835373940 \mathrm{~d}+02$ $3.593880610292548417862187 d+02$ $3.786171415077789828663518 d+02$ $3.983464864945479141401659 d+02$
$8.8575 d-01$ $2.5341 d-01$ $3.7166 \mathrm{~d}-02$ $4.2771 d-03$ 4. $3128 \mathrm{~d}-04$ $3.9986 d-05$ 3. $4973 d-06$ $2.9303 d-07$ 2. $3754 \mathrm{~d}-08$ $1.8758 \mathrm{~d}-09$ $1.4500 \mathrm{~d}-10$ $1.1012 \mathrm{~d}-11$ $8.2392 d-13$ $6.0862 \mathrm{~d}-14$ 4. $4465 \mathrm{~d}-15$ 3. $2172 \mathrm{~d}-16$ 2.3081d-17 1. $6433 d-18$ $1.1621 \mathrm{~d}-19$ $8.1686 \mathrm{~d}-21$ $5.7101 d-22$ $3.9715 d-23$ 2.7497d-24 $1.8958 \mathrm{~d}-25$ 1. $3020 \mathrm{~d}-26$ $8.9102 \mathrm{~d}-28$ $6.0775 \mathrm{~d}-29$ $4.1327 d-30$ $2.8022 \mathrm{~d}-31$ $1.8950 \mathrm{~d}-32$ $1.2783 d-33$ $8.6024 d-35$ $5.7764 d-36$ $3.8708 d-37$ $2.5888 \mathrm{~d}-38$ $1.7282 \mathrm{~d}-39$ $1.1516 \mathrm{~d}-40$ $7.6617 \mathrm{~d}-42$ $5.0892 d-43$ $3.3754 d-44$ 
Appendix A2: Recursion coefficients $\alpha_{k}, \beta_{k}$ and error constants $\gamma_{k}$ for orthogonal polynomials $\pi_{k}\left(\cdot ; \varphi_{r} d t\right), r=1,2$.

TABLE 3

Recursion coefficients and error constants in the case $r=1$

k

a $\operatorname{lpha}(\mathrm{k})$ beta $(k)$

$6.931471805599453094172321 d-01$

$1.193356045789508659178946 \mathrm{~d}+00$ $4.191806424549042451590083 d+00$ $9.215367385434641976544932 d+00$ $1.623913244828318399783906 d+01$ 2. $526147321685195747738024 d+01$ $3.628235105123771646231038 d+01$ $4.930193771925747304811208 d+01$ $6.432041306075684261406964 d+01$ $8.133793106899114661883749 d+01$ $1.003546181232714737844363 d+02$ $1.213705773374930439179113 d+02$ $1.443858933064312247341199 d+02$ $1.694006360695312561343422 d+02$ $1.964148642010325651995636 d+02$ 2. $254286271736712212879007 d+02$ $2.564419671643648677565733 d+02$ $2.894549204416899668238851 d+02$ 3. $244675184414076026002832 d+02$ $3.614797886084236427881509 d+02$ $4.004917550625525317022511 d+02$ $4.415034391301419740031332 d+02$ $4.845148597725808406593359 d+02$ $5.295260339347629377708534 d+02$ $5.765369768308248977038621 d+02$ $6.255477021802805949656030 d+02$ 6. $765582224045888408376874 d+02$ $7.295685487919013322017815 d+02$ $7.845786916360230644686497 d+02$ $8.415886603543216590530846 d+02$ $9.005984635883343186160464 d+02$ $9.616081092900618200644516 d+02$ $1.024617604796350530317238 d+03$ $1.089626956893303904655591 d+03$ $1.156636171872303425240697 d+03$ $1.225645255578932558332990 d+03$ 1. $296654213455868932332327 d+03$ $1.369663050580626665464266 \mathrm{~d}+03$ $1.444671771698882809763324 d+03$ $1.521680381254001740382063 d+03$ $\operatorname{gamma}(k)$

$6.9315 d-01$

$4.1359 \mathrm{~d}-01$

$1.4447 \mathrm{~d}-01$

4. $4379 d-02$

1. $2869 \mathrm{~d}-02$

3. $6122 \mathrm{~d}-03$

$9.9286 d-04$

2. $6896 \mathrm{~d}-04$

7. $2081 \mathrm{~d}-05$

1. $9160 \mathrm{~d}-05$

5.0599d-06

1.3293d-06

3. $4770 d-07$

$9.0616 d-08$

2. $3543 d-08$

$6.1002 \mathrm{~d}-09$

1. 5770d-09

4.0683d-10

$1.0476 \mathrm{~d}-10$

2. $6935 d-11$

6. $9148 d-12$

1. $7729 \mathrm{~d}-12$

4. 5401 d-1 3

$1.1614 \mathrm{~d}-13$

2.9681d-14

$7.5782 d-15$

1.9333d-1 5

$4.9283 d-16$

1. $2554 \mathrm{~d}-16$

3. $1958 \mathrm{~d}-17$

8.1303d-18

$2.0672 d-18$

5. $2532 d-19$

$1.3343 \mathrm{~d}-19$

3. $3873 d-20$

8. $5956 d-21$

$2.1803 d-21$

$5.5280 d-22$

1. $4011 d-22$

3. $5498 d-23$ 


\section{TABLE 4}

Recursion coefficients and error constants in the case $r=2$

$6.695404638538438232387227 \mathrm{~d}-01$ $1.664686133009829680011075 d+00$ $2.619431841015758476604192 d+00$ $3.593865366974072299756279 d+00$ $4.579560611244422509231409 d+00$ $5.570393244889081013183465 d+00$ $6.563863856463274821282662 d+00$ $7.558890435710582391017287 \mathrm{~d}+00$ $8.554931506945101735350174 d+00$ $9.551680424073512844025433 d+00$ $1.054894749426849736758553 d+01$ $1.154660774499827949400336 \mathrm{~d}+01$ 1. $254457487195045007921430 d+01$ l. $354278703591851122550789 d+01$ $1.454119858463881673190978 d+01$ $1.553977497041409480722936 d+01$ $1.653848949597723105885520 d+01$ $1.753732115701126023268357 \mathrm{~d}+01$ $1.853625316885666471251907 \mathrm{~d}+01$ $1.953527193452852551824561 d+01$ $2.053436630566769258684606 d+01$ $2.153352704286241009913773 \mathrm{~d}+01$ 2. $253274641468520667332283 d+01$ $2.353201789515210436373844 d+01$ $2.453133593225018788013906 d+0$ $2.553069576859866483809419 d+01$ $2.653009330090433519953643 d+01$ $2.752952496866371491931937 d+01$ $2.852898766517794626792057 d+01$ 2.952847866577761073760216d+01 $3.052799556945594577910743 d+01$ $3.152753625104640930157417 d+0$ 3. $252709882176421757703103 d+01$ 3.352668159643583823594838d+01 $3.452628306611646621975720 d+01$ 3. $552590187507868194832644 d+01$ 3. $652553680137070829557449 d+01$ $3.752518674030767687262801 d+01$ 3. $852485069038683713846996 d+0$ $3.952452774121695980565398 d+01$
1. $931471805599453094172321 d-01$ 3.705278710851684856806622d-01 1. $154907853652601365365824 d+00$ $2.416782871937569027723998 d+00$ $4.178983363598481071166374 d+00$ $6.442373341823139342338233 d+00$ $9.205824543736968972236729 d+00$ $1.246885990410737186232674 d+01$ $1.623136041040844992228737 d+01$ $2.049332827585098989839084 d+01$ $2.525480038308609252223979 d+01$ $3.051582056896307516793924 d+01$ 3. $627643102788570772578227 d+01$ 4. $253666988039415434188650 d+01$ 4. $929657079160519734344687 d+01$ $5.655616326612624756002118 d+01$ $6.431547313435234317081962 d+01$ 7.257452305464815707363551d+01 $8.133333297094291496385707 d+01$ $9.059192050950994107419572 d+01$ $1.003503013155485508754740 d+02$ $1.106084893360278480033078 d+02$ 1. $213664970566454120865567 d+02$ 1. $326243357004168602197873 d+02$ l. $443820153944878713298334 d+02$ l. $566395453107226933490024 d+02$ $1.693969337846574069748673 d+02$ l. $826541884165723339055932 \mathrm{~d}+02$ $1.964113161577435259558123 d+02$ $2.106683233843655691641098 d+02$ 2. $254252159611781753560632 d+02$ 2. $406819992964582304786437 d+02$ $2.564386783897402174805713 d+02$ $2.726952578733870106688784 d+02$ $2.894517420489383379229000 d+02$ $3.067081349190064005746629 d+02$ 3. $244644402153598017416908 d+02$ $3.427206614237321739508610 d+02$ $3.614768018058060537519819 \mathrm{~d}+02$ 3. $807328644187519319245180 d+02$
$1.9315 d-01$ 3. $5783 \mathrm{~d}-02$ $3.4439 \mathrm{~d}-03$ $2.7744 \mathrm{~d}-04$ $2.0704 d-05$ $1.4820 \mathrm{~d}-06$ $1.0336 \mathrm{~d}-07$ $7.0810 d-09$ 4.7889d-10 3. 2072d-11 $2.1315 d-12$ 1. $4079 d-13$ $9.2524 d-15$ $6.0549 d-16$ $3.9482 \mathrm{~d}-17$ $2.5666 \mathrm{~d}-18$ $1.6640 \mathrm{~d}-19$ $1.0764 d-20$ $6.9479 d-22$ $4.4767 d-23$ $2.8797 d-24$ $1.8497 \mathrm{~d}-25$ $1.1865 d-26$ 7. $6022 d-28$ 4. $8653 d-29$ 3. $1106 \mathrm{~d}-30$ $1.9869 d-31$ 1. $2681 d-32$ 8.0864d-34 $5.1529 d-35$ 3. $2813 d-36$ $2.0882 d-37$ 1. $3281 d-38$ 8. $4422 d-40$ 5. $3635 d-41$ 3. $4059 \mathrm{~d}-42$ $2.1617 d-43$ $1.3715 d-44$ $8.6975 d-46$ 5. $5135 d-47$ 
Department of Computer Sciences

Purdue University

West Lafayette, Indiana 47907

Faculty of Electronic Engineering

Department of Mathematics

University of Niš

18000 Niš, Yugoslavia

1. J. S. Blakemore, Solid State Physics, 2nd ed., Saunders, Philadelphia, Pa., 1974.

2. W. GaUtsCHI, “Algorithm 542-Incomplete gamma functions," ACM Trans. Math. Software, v. 5, 1979, pp. 482-489.

3. W. GautschI, "A survey of Gauss-Christoffel quadrature formulae," in E. B. Christoffel-The Influence of his Work in Mathematics and the Physical Sciences (P. L. Butzer and F. Fehér, eds.), Birkhäuser Verlag, Basel, 1981, pp. 72-147.

4. W. GaUTSCHI, “On generating orthogonal polynomials," SIAM J. Sci. Statist. Comput., v. 3, 1982, pp. 289-317.

5. J. F. HART et al., Computer Approximations, Wiley, New York-London-Sydney, 1968.

6. A. MCLellan IV, "Tables of the Riemann zeta function and related functions," Math. Comp., v. 22, 1968, Review 69, pp. 687-688.

7. S. S. Mitra \& N. E. MASSA, "Lattice vibrations in semiconductors," Chapter 3 in: Band Theory and Transport Properties (W. Paul, ed.), pp. 81-192. Handbook on Semiconductors (T. S. Moss, ed.), Vol. 1. North-Holland, Amsterdam, 1982.

8. F. REIF, Fundamentals of Statistical and Thermal Physics, McGraw-Hill, New York, 1965.

9. R. A. Smith, Semiconductors, 2nd ed., Cambridge Univ. Press, Cambridge, 1978.

10. R. J. Van Overstraeten, H. J. DeMan \& R. P. Mertens, “Transport equations in heavy doped silicon," IEEE Trans. Electron Dev., v. ED-20, 1973, pp. 290-298. 


\title{
Supplement to \\ Gaussian Quadrature Involving Einstein and Fermi Functions with an Application to Summation of Series
}

\author{
By Walter Gautschi and Gradimir V. Milovanović
}

TABLE 5. Nodes and weights for $n$-point Gaussian quadrature with respect to the weight function $\varepsilon_{1}(t)=t /\left(e^{t}-1\right)$ on $(0, \infty), \quad n=5(5) 40$. $1.789519270251396059078587 \mathrm{~d}+00$ $4.317073039784563286568702 d+00$ $8.124316435674601205701546 \mathrm{~d}+00$ $1.399259503591182245471045 d+01$
$1.712764587800172362970467 \mathrm{~d}-0$ $8.916728564071628155969295 d-01$
$2.154696241995276926728150 d+00$ $3.940962194432075308546014 \mathrm{~d}+00$ $6.273054978120200583717756 \mathrm{~d}+00$ 9. $219833208404748987213936 d+00$ 1. $289612902426177067770092 d+01$ $1.749262020229698453921089 \mathrm{~d}+01$ $2.337506876689075787489480 d+0$ $3.148092990870547794581304 \mathrm{~d}+0$

$1.122689742882840428560134 \mathrm{~d}-01$ $5.872942570832169304369100 d-01$ $1.427134594172779508922935 d+00$ $2.616230204390486070960179 d+00$ $4.148505367683641117556727 \mathrm{~d}+00$ $6.033516951668504802085092 \mathrm{~d}+00$ $8.294299990936086527007386 \mathrm{~d}+00$ $1.096384343614386146477958 d+01$ $1.408587628929527609167703 d+01$ $1.771999691156769233871477 \mathrm{~d}+0$ $2.195126297803758949286577 \mathrm{~d}+01$ $2.690847821599200481688143 d+01$ $3.280454835374218314720542 d+0$ 4.00464897407709594053175ld+0 $4.967641464169027902025467 d+01$ we ight (n)

$7.413801893864949064648418 d-01$ $7.112793968534230324727631 d-01$ 1. $810544213015127812690667 d-01$ $1.113156581019995660932093 d-02$ $8.849349659575965642269187 d-05$

4. $017581983871970550764847 d-01$ $6.178151502068598877652466 \mathrm{~d}-01$ $4.309238491671243158403736 \mathrm{~d}-01$ $1.601831853477292223382136 \mathrm{~d}-01$ $3.111600156831707548706688 d-02$ $3.002950279906314058385099 \mathrm{~d}-03$ 1. $324400356318608169220646 \mathrm{~d}-04$ 2. $280734015322767264428365 d-06$ $1.111475587288852659683946 \mathrm{~d}-08$ $6.689509433931585817256954 d-12$ $4.852206727225164096245477 d-01$ $4.581586361360587417881249 d-01$ $2.806056660239435321238224 d-01$ $1.135140668971388075471236 \mathrm{~d}-01$ $2.998304918534276590192265 d-02$ $5.098666065215486743990180 d-03$ $5.482040128082603574763532 d-04$ $3.618564942449709026477206 \mathrm{~d}-05$ 1.398222595231963340037460d-06 $2.936603420052541839075062 d-08$ $2.982123035751269147999240 d-10$ $1.203079310277072656860401 \mathrm{~d}-12$ 1. $324544206896025265830478 d-15$ $1.546415245123910288693076 d-19$ 

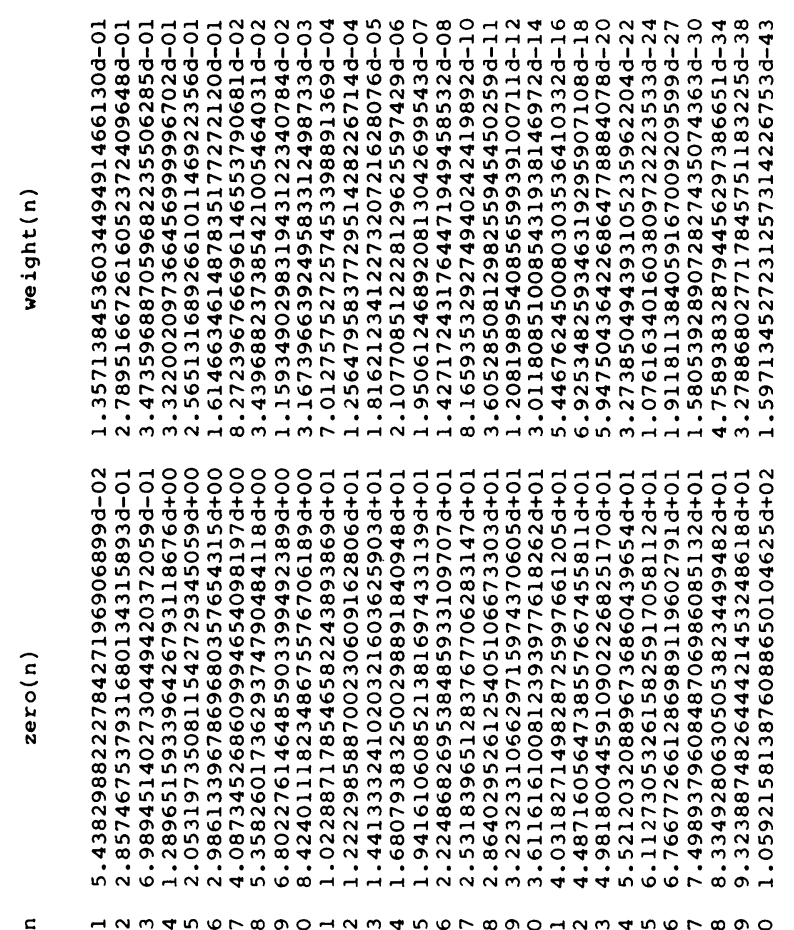

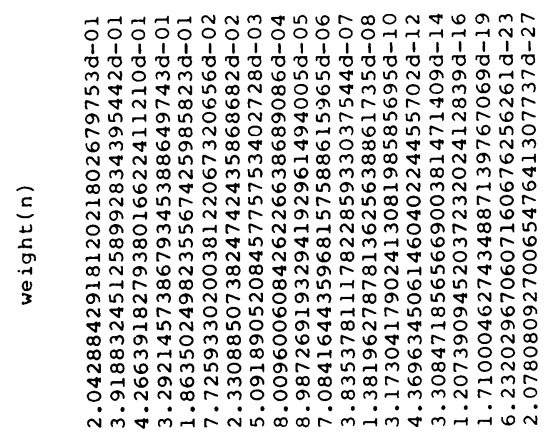

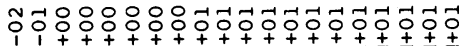
1 0

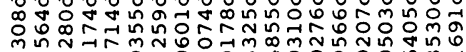

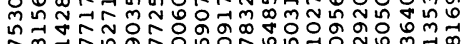
解行

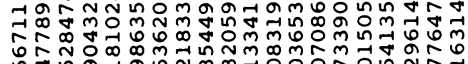

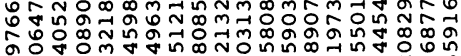

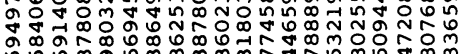

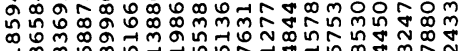

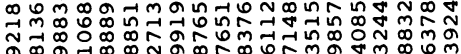
ลै

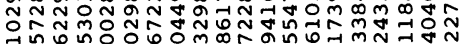

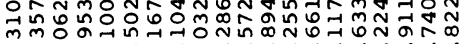

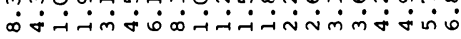

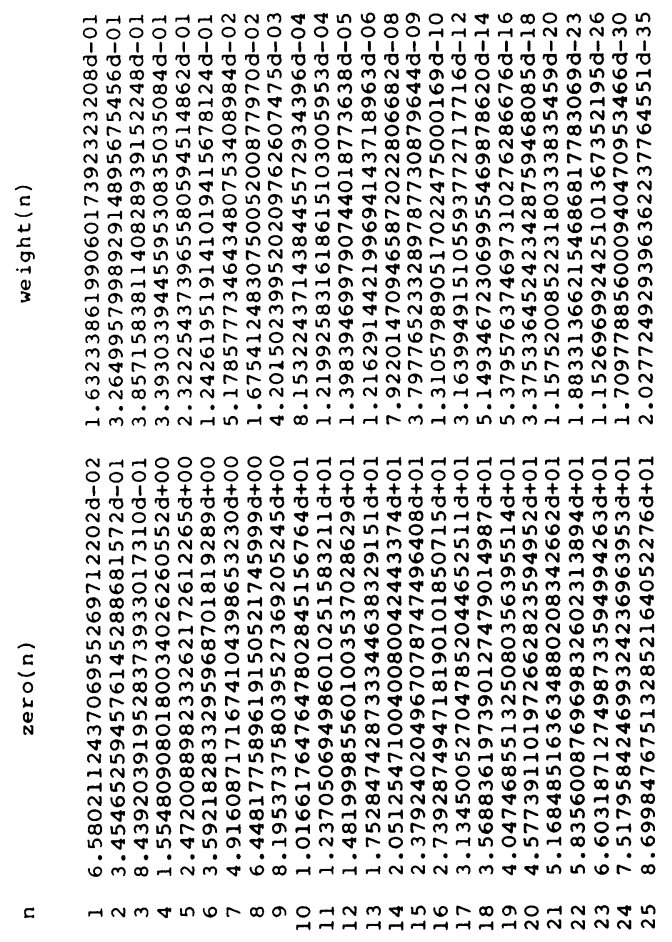




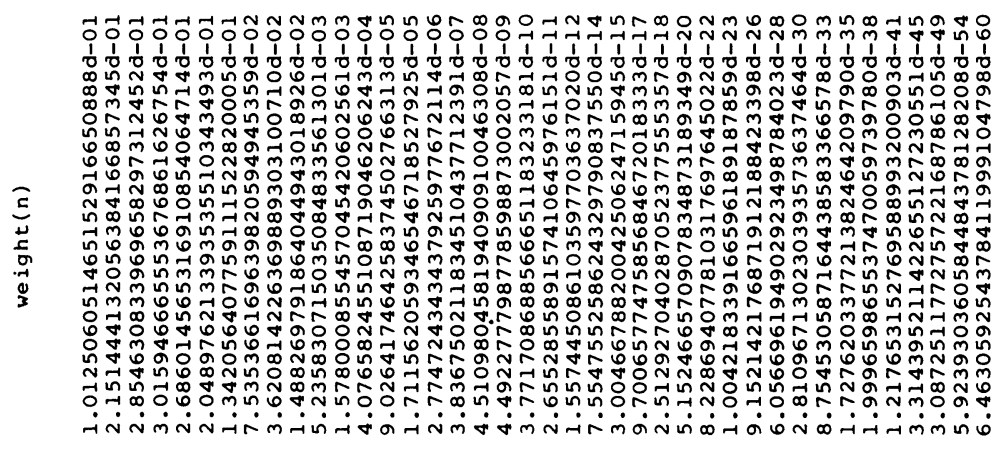

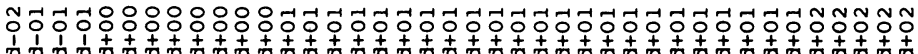

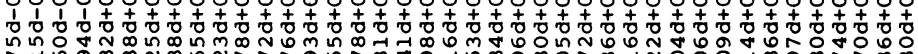

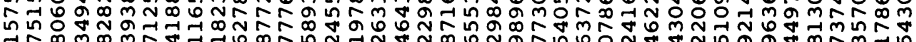
7.

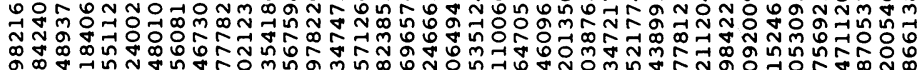
三

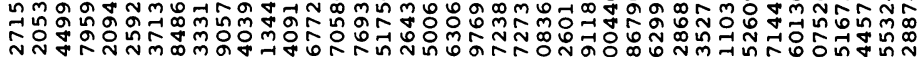

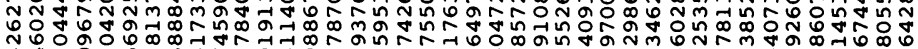

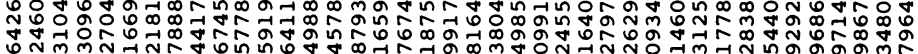

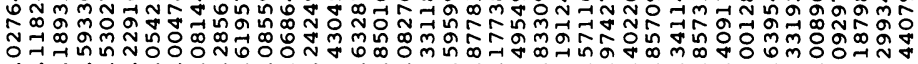

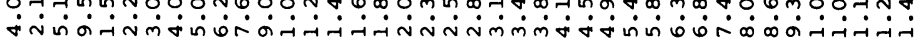
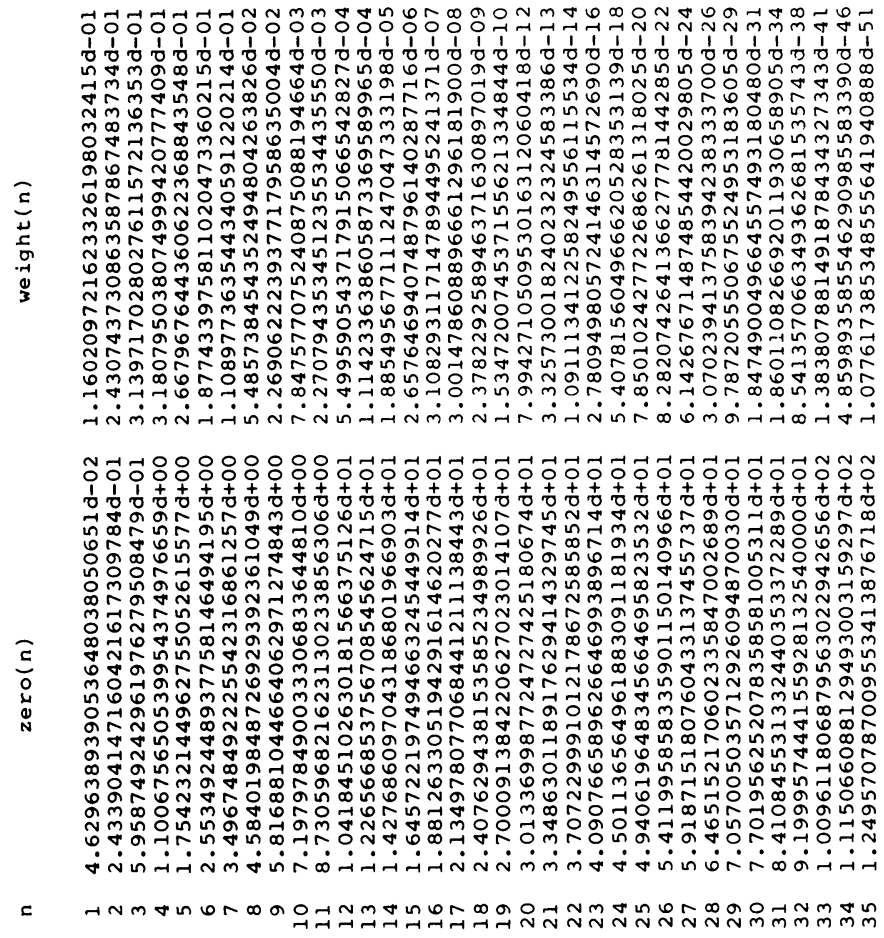

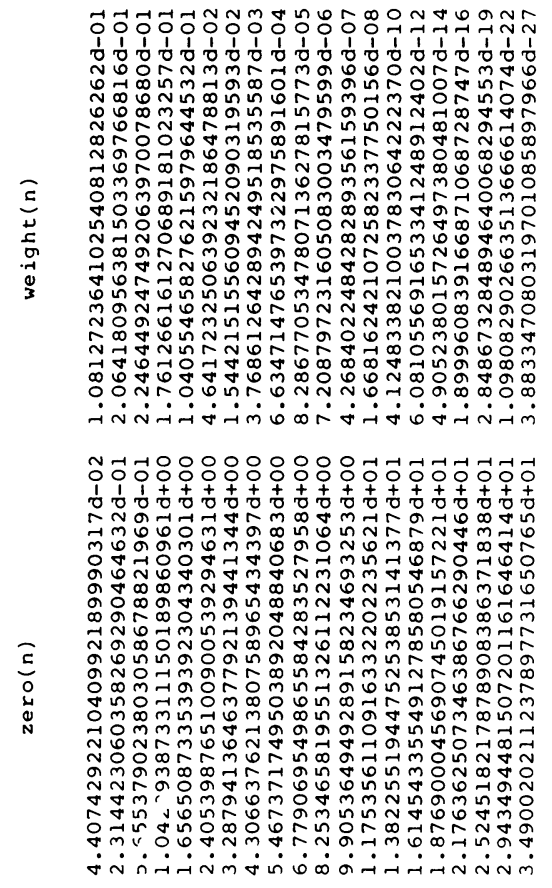

c

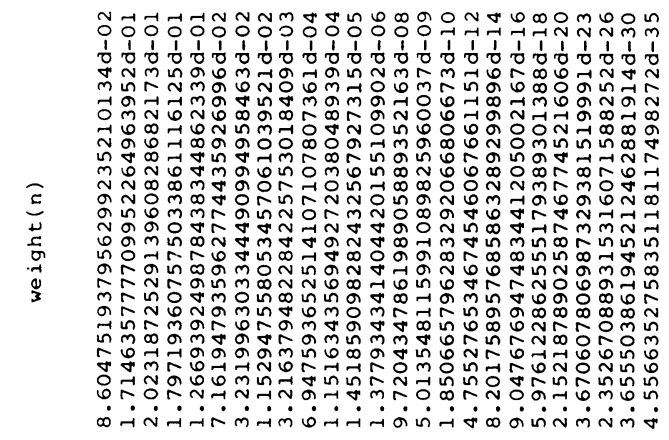

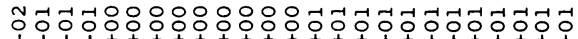

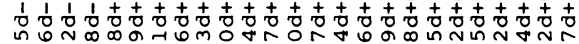
o

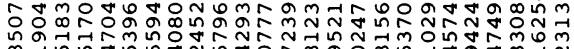
œ

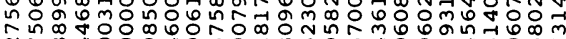
N

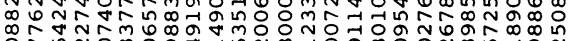

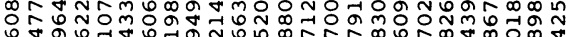

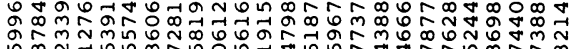

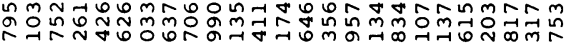
क

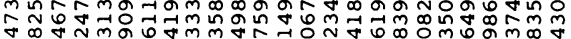

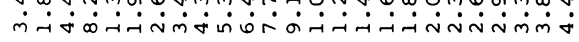

c -Nmon

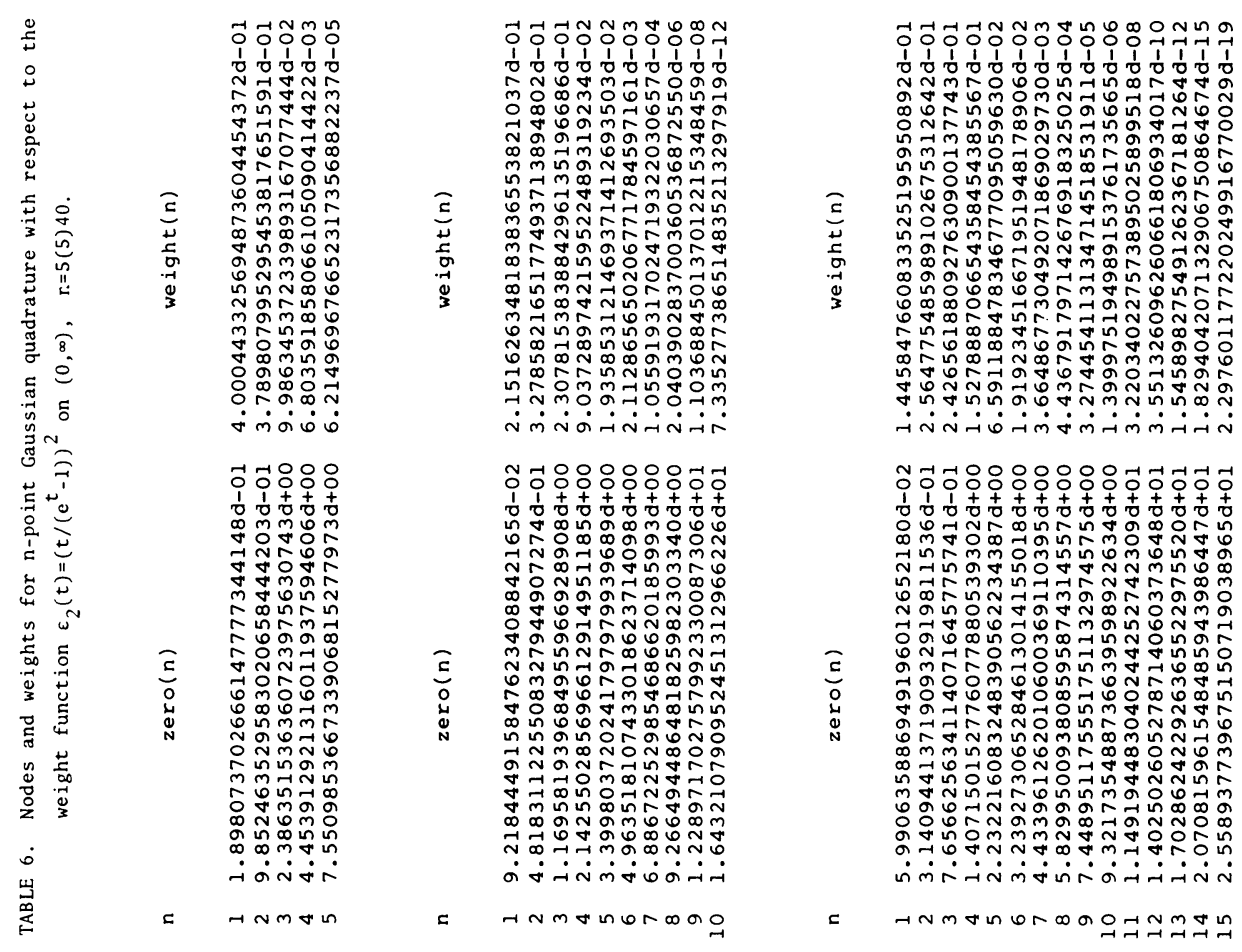




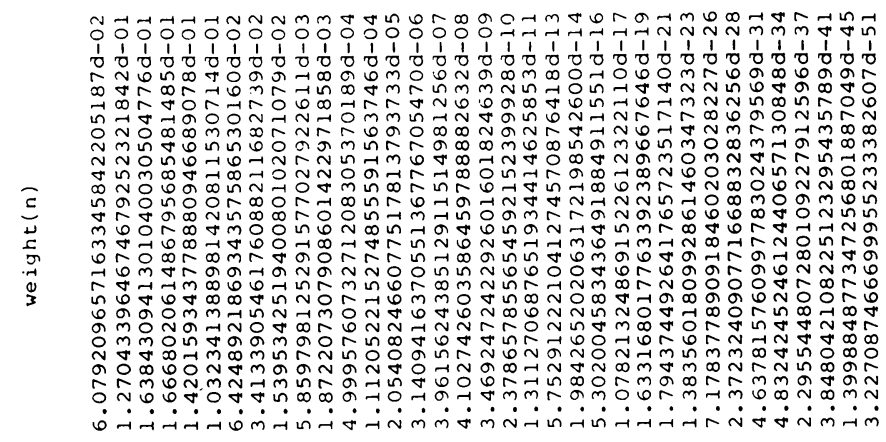

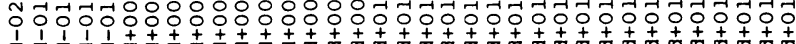

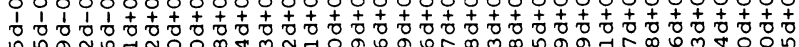

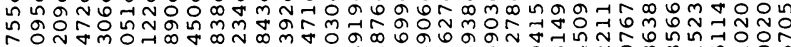

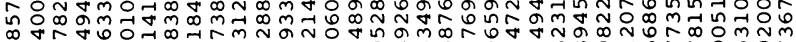
Dơ

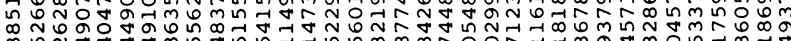

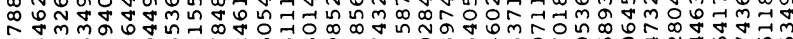

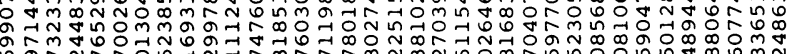

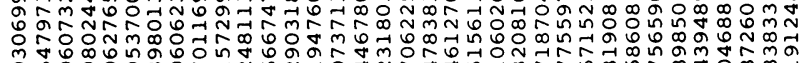

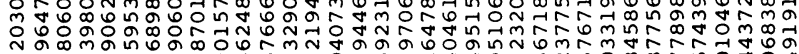

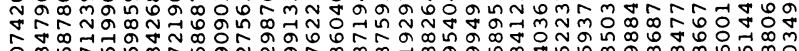

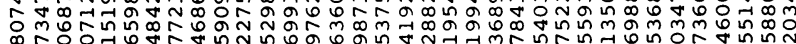

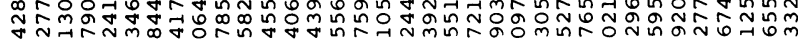

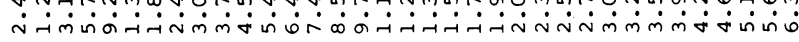
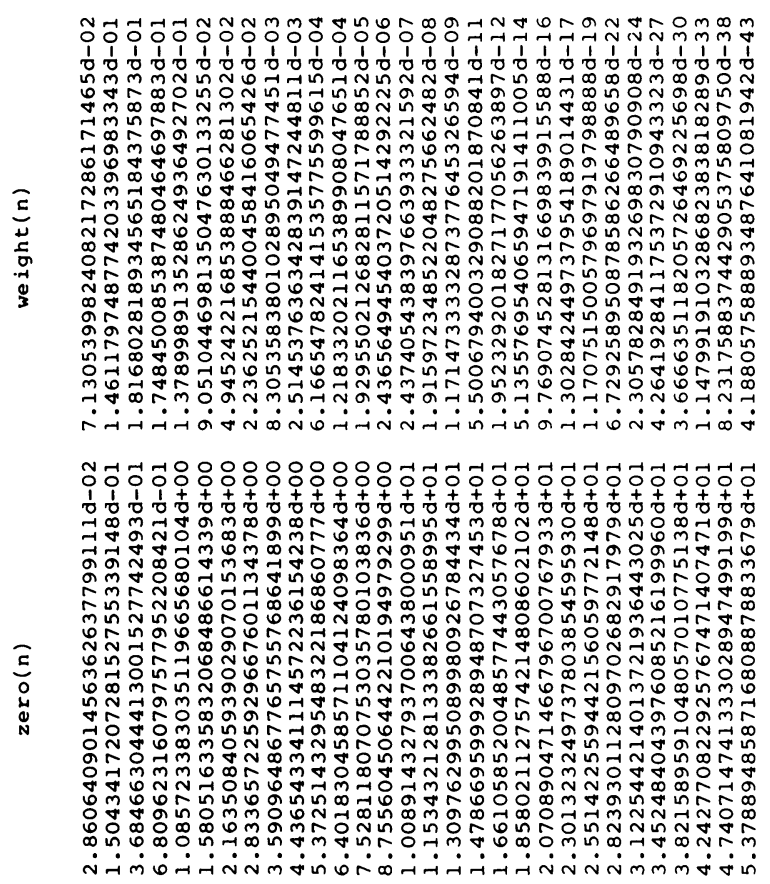


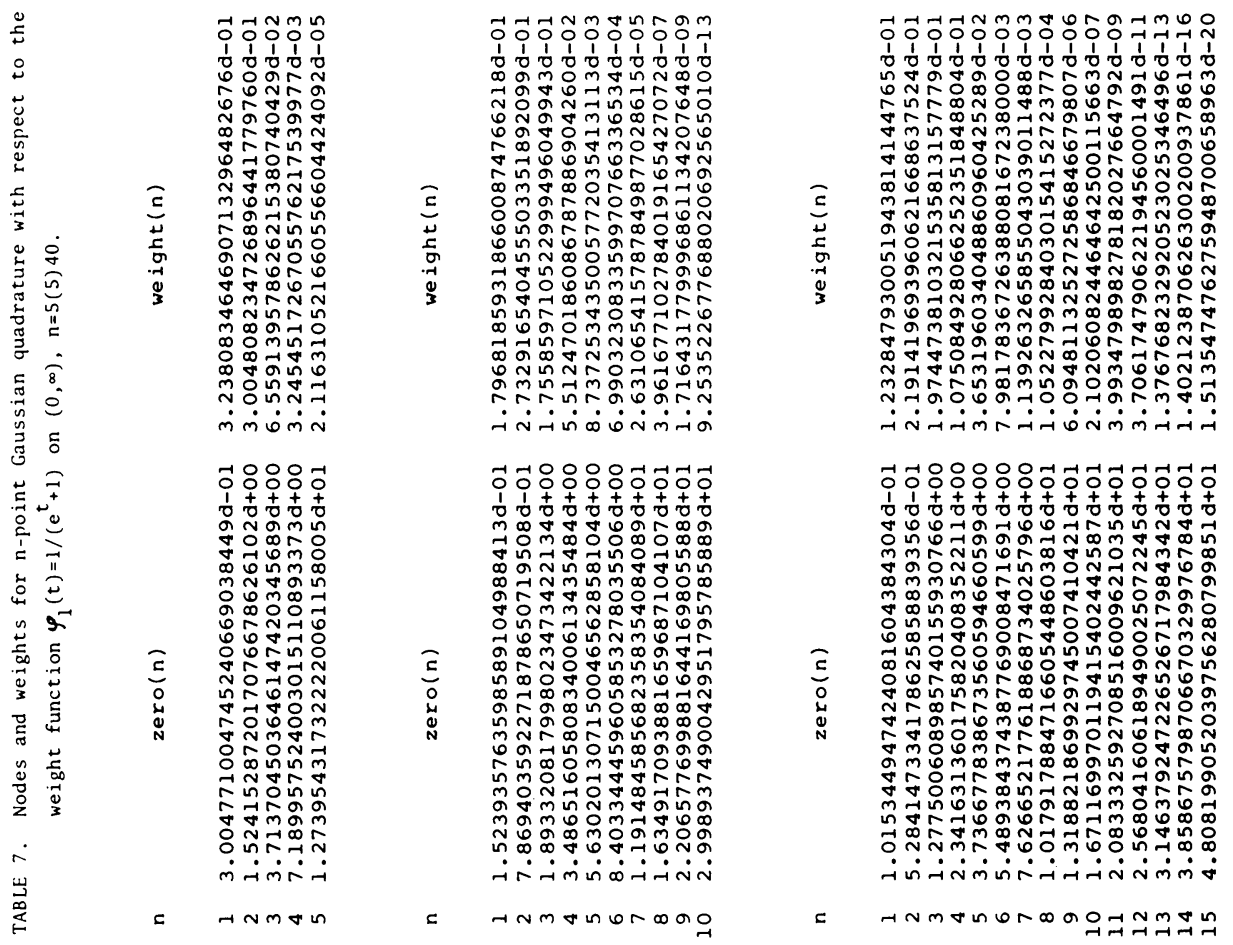
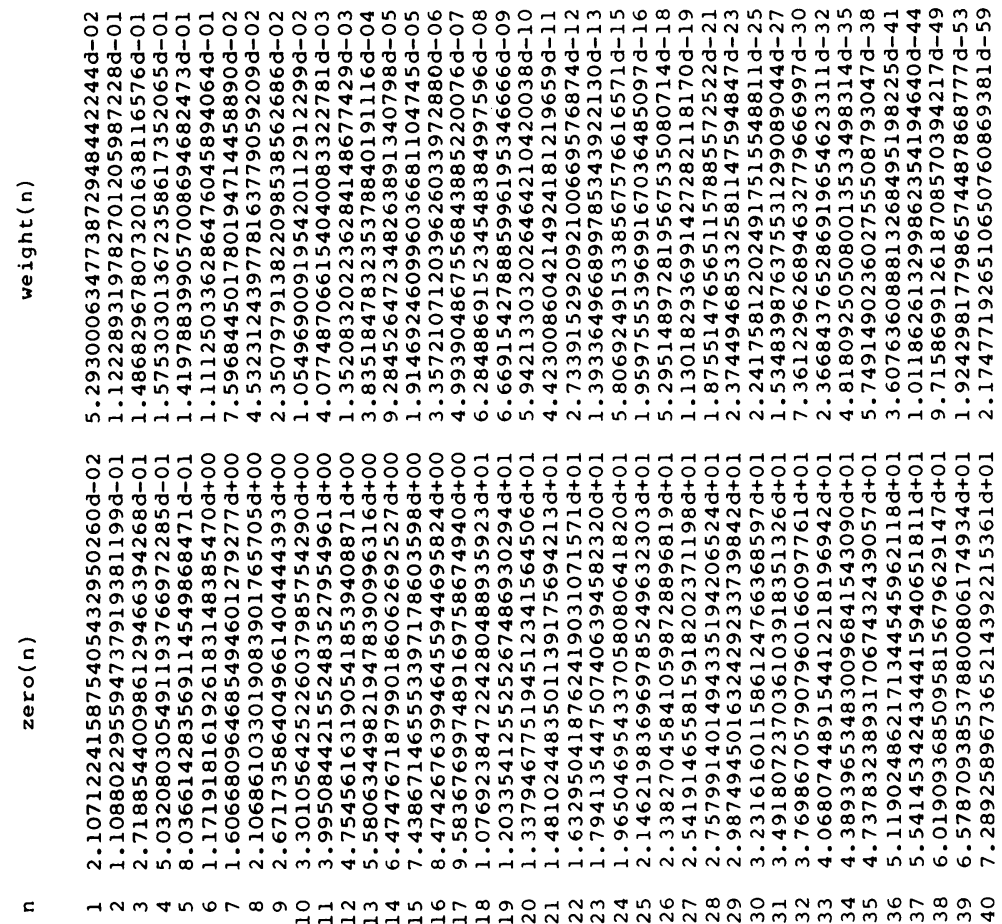

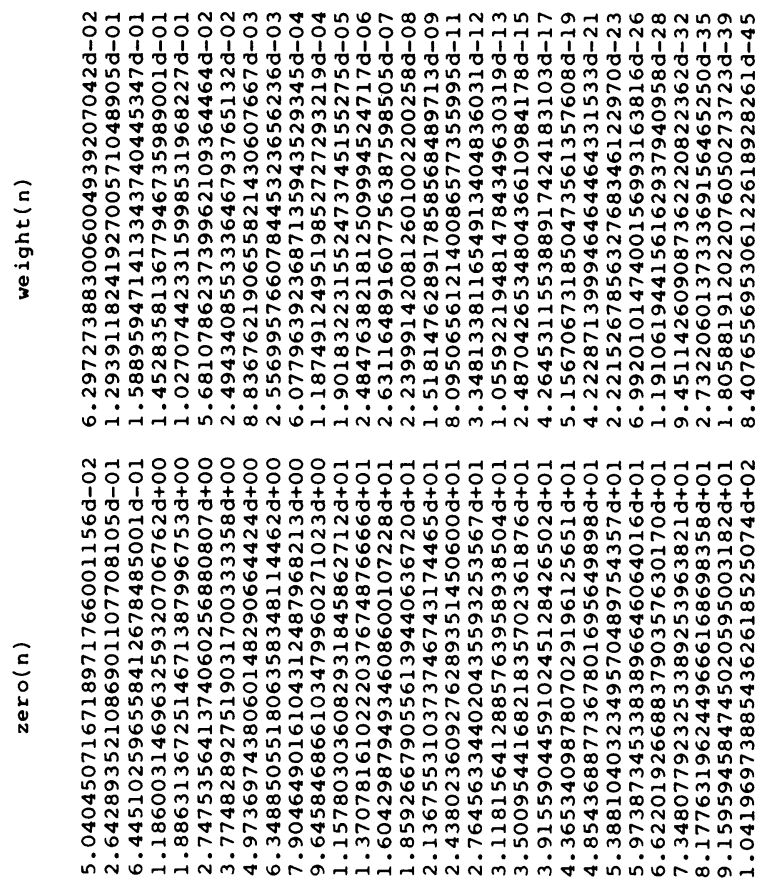

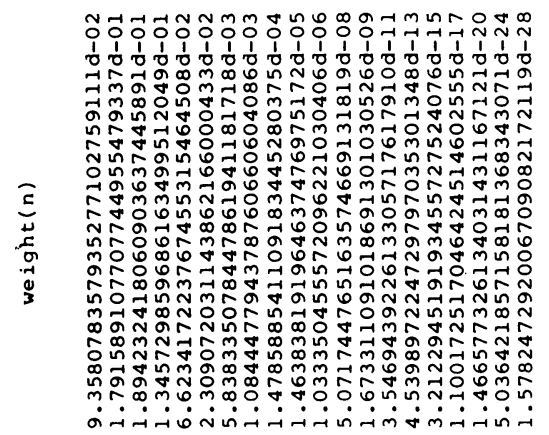

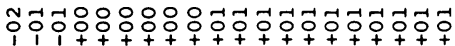

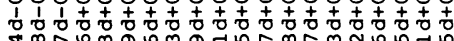

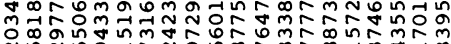

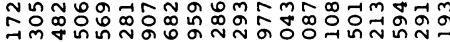

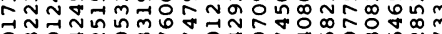

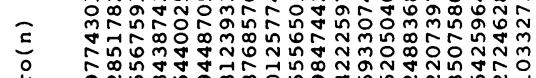

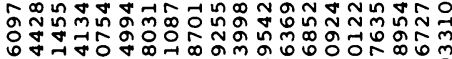

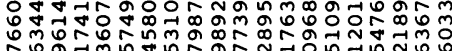

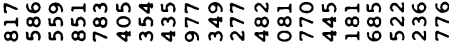

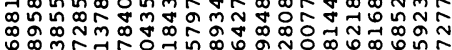

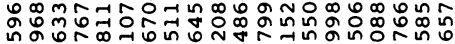

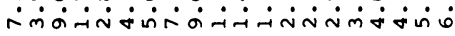

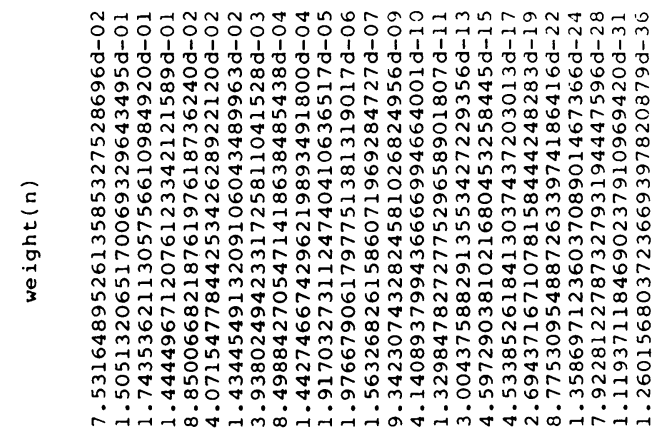

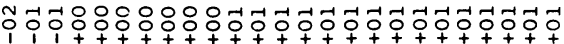

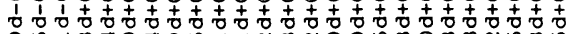

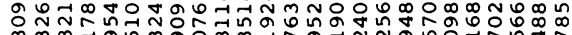

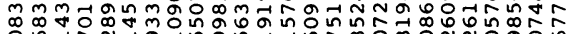

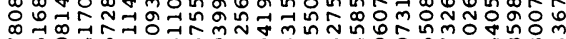
๓

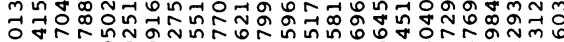

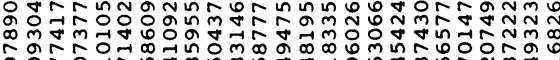

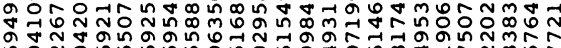

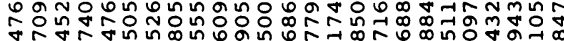

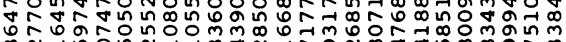

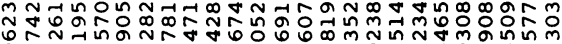

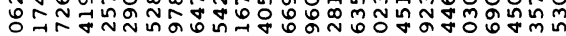

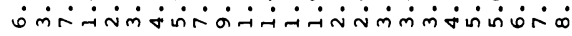



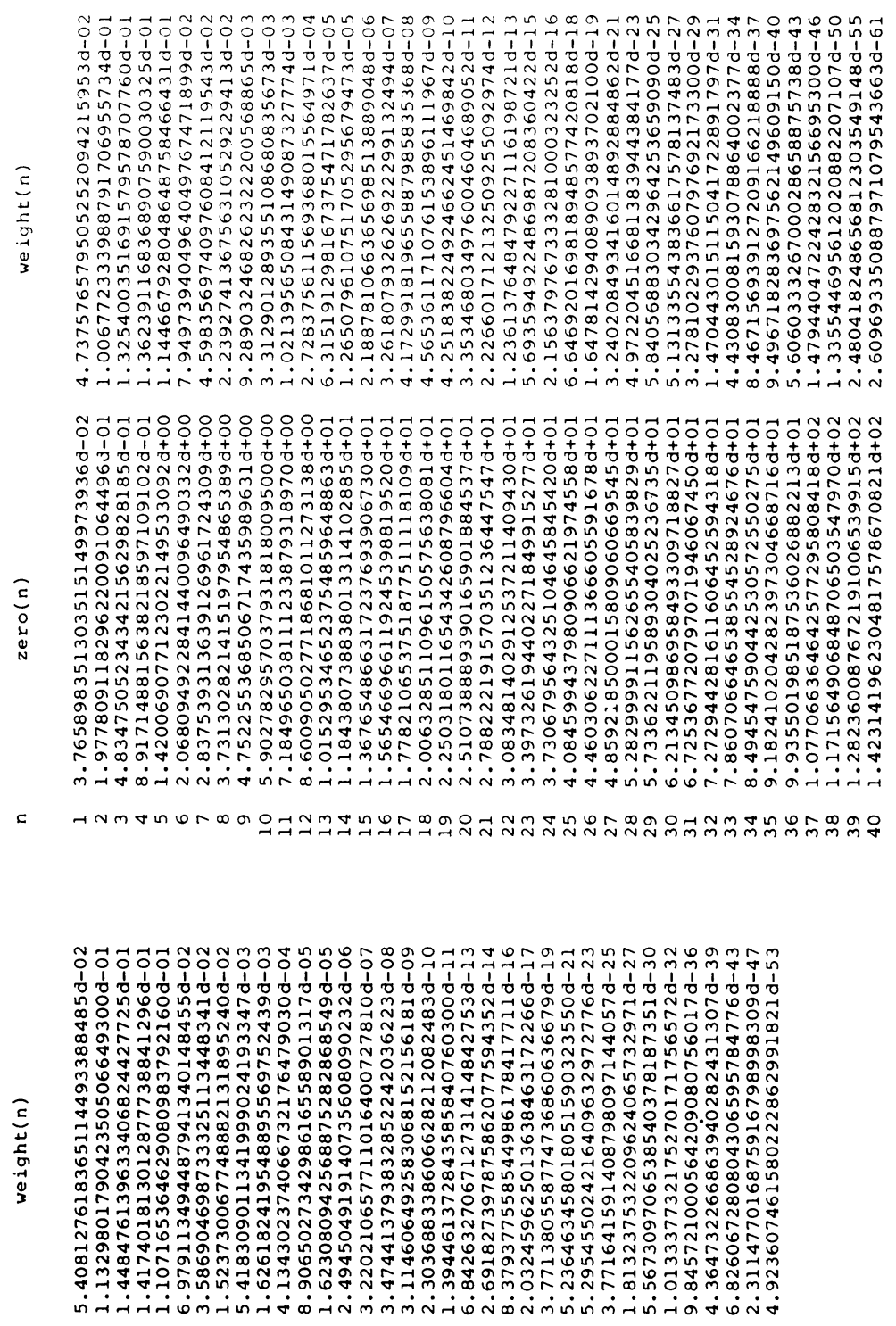

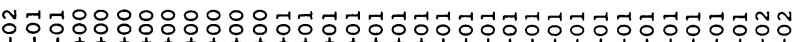
10

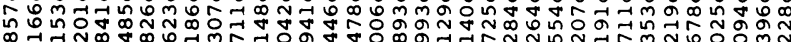
等 जैं

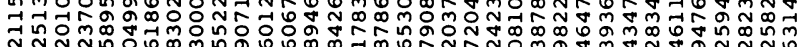
NNㅜㄴ ỡ లై

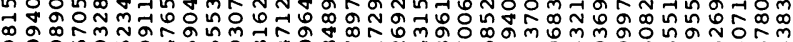

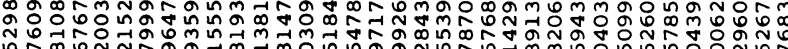
Now

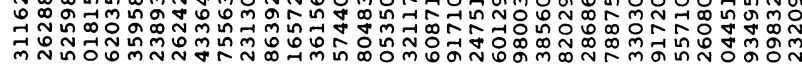
अं 


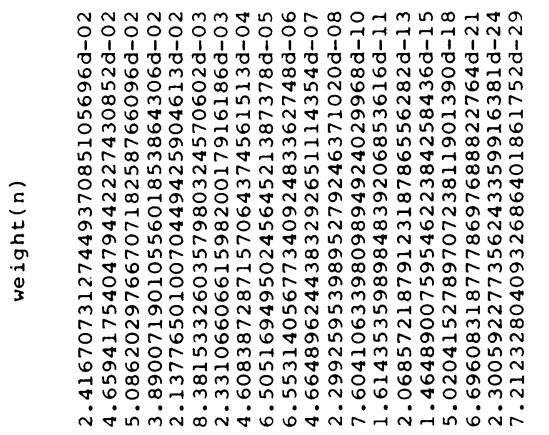

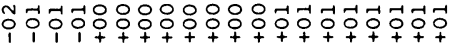
10

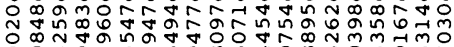

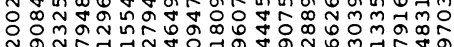

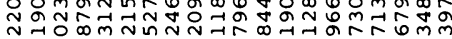

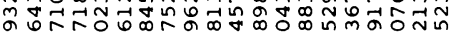
ฟ Nิก ₹

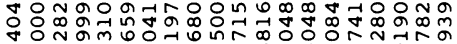

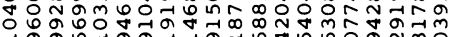

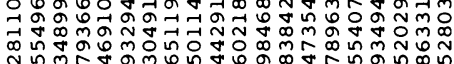
认ñ

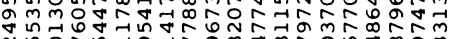

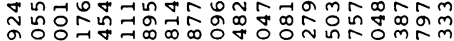

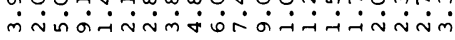

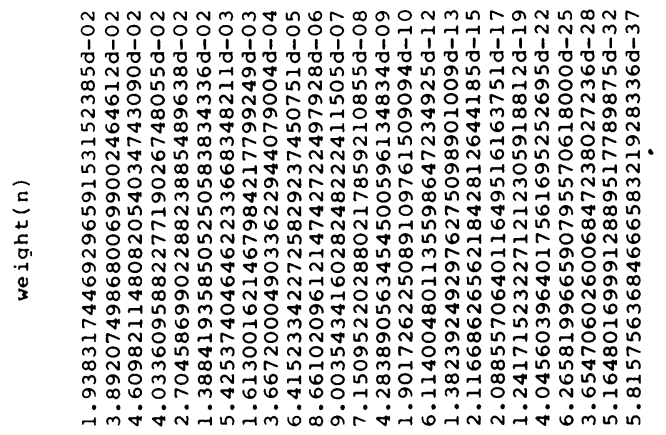

ฮั

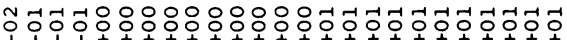
ำ ำ-1

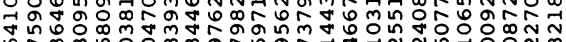

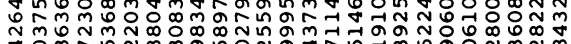

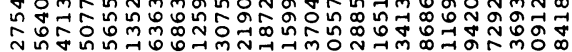

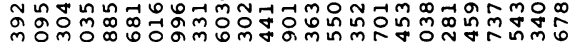
மํำ

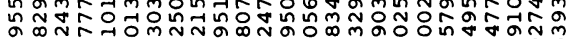
న.

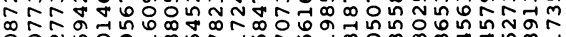

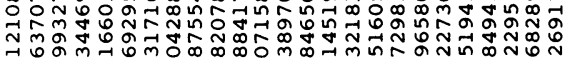

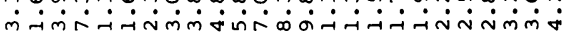

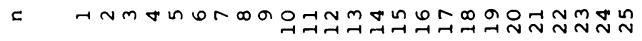

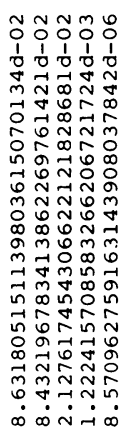

구유요

ind

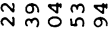

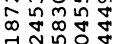

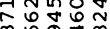

每

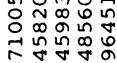

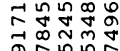

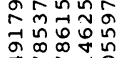

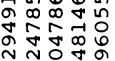

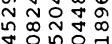

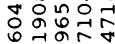

-i $\dot{-1} \dot{-} \dot{0}$

c

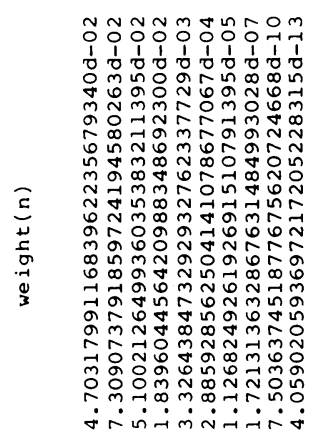

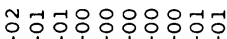

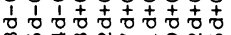

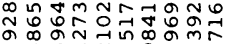

ชิ ฒNำ

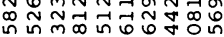

คํำ

c

人ูนำ

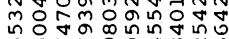

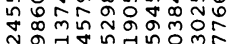

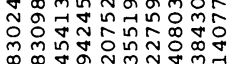

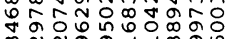

ल) त N

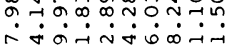

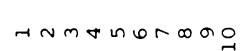

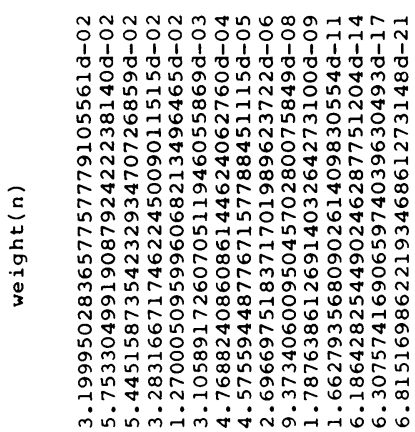

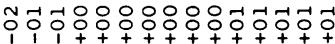
1 : N

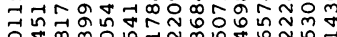

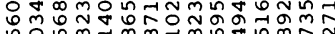

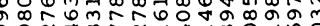

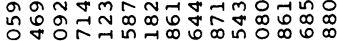

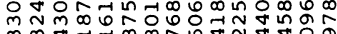

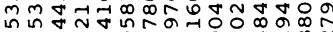

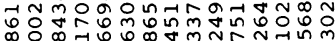
等

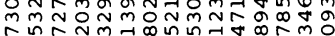

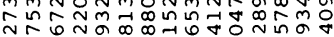

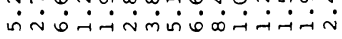

c TNmon 

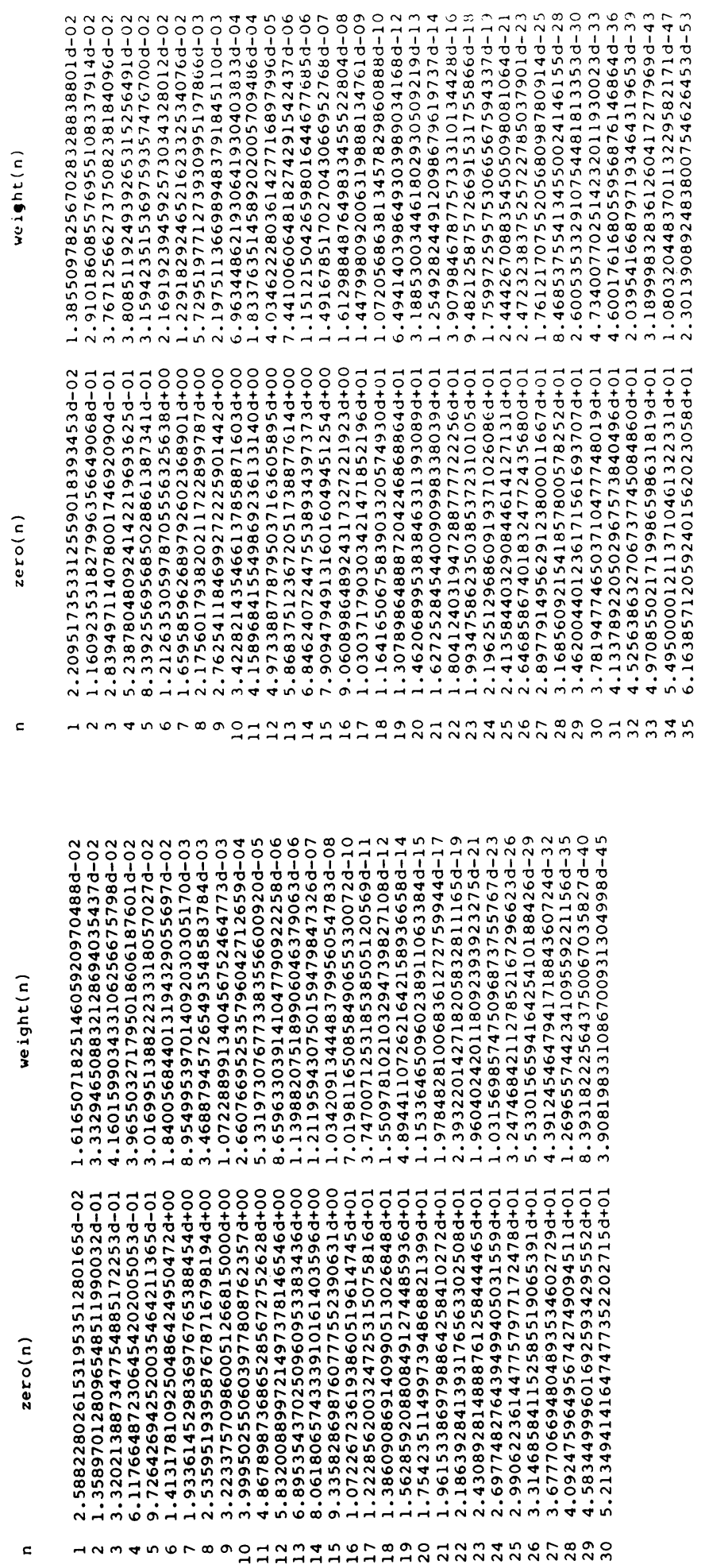

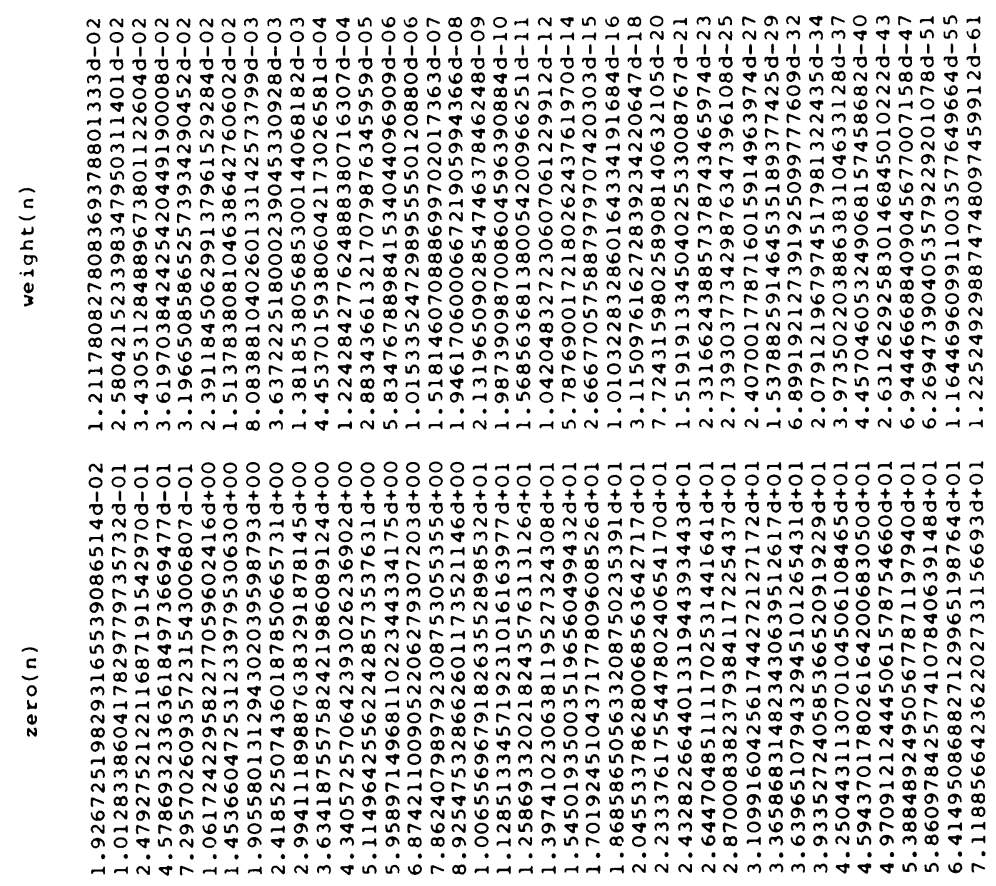

c

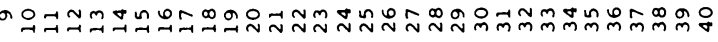

